\title{
Revision of the Structure and Total Synthesis of Topsentin C
}

\author{
Nikita E. Golantsov* \\ Alexey A. Festa \\ Alexey V. Varlamov \\ Leonid G. Voskressensky
}

Peoples' Friendship University of Russia (RUDN University), 6 Miklukho-Maklaya St., Moscow 117198, Russian Federation golantsov_ne@pfur.ru

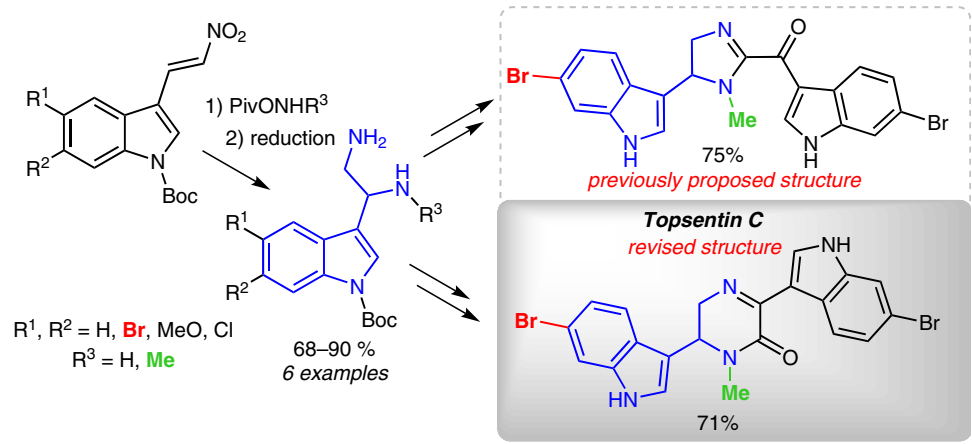

Received: 22.11 .2016

Accepted after revision: 01.02.2017

Published online: 23.02 .2017

DOI: 10.1055/s-0036-1588731; Art ID: ss-2016-n0804-op

License terms: (c)

Abstract An efficient synthetic approach to access (indol-3-yl)ethane1,2-diamines with a protecting group at the indole $\mathrm{N}$ atom from readily available 3-(2-nitrovinyl)indoles is reported. This approach includes solvent-free conjugate addition of $\mathrm{O}$-pivaloylhydroxylamines to 1 -Boc-3(2-nitrovinyl)indoles followed by mild reduction of the adducts. The obtained (indol-3-yl)ethane-1,2-diamines are convenient synthetic precursors for several classes of marine alkaloids. The first total synthesis of racemic topsentin C, a secondary metabolite from Hexadella sp., based on this approach is reported. The initially proposed structure for topsentin $C$ has been revised.

Key words bisindole alkaloid, topsentin, hamacanthin, spongotine, diamine, hydroxylamine

Secondary metabolites from marine invertebrates continue to be an attractive research topic because new structures and compounds with useful biological activity can be discovered. ${ }^{1} \mathrm{~A}$ whole series of alkaloids containing the (indol-3-yl)ethane-1,2-diamine moiety in their structures and their aromatized derivatives were isolated from deep-water sponges in the last 30 years. ${ }^{2}$ In particular, spongotines (1) and topsentins (2) contain two indoles connected through imidazoline or imidazole linker. Two indole substituents in the structures of hamacanthins (3) and dragmacidins (4) are bonded to dihydropyrazinone and piperazine rings, respectively (Figure 1). The (indol-3-yl)ethane-1,2-diamine moiety in several alkaloids of the examined group contains one or two methyl groups; for example, dragmacidins A and B (4) and topsentin C. The latter compound was isolated from Hexadella sp., and its structure was assigned to imidazoline derivative $\mathbf{5 a}$ (Figure 2). ${ }^{3}$ Furthermore, the alkaloids could contain one or more $\mathrm{Br}$ atoms, which in general is characteristic of marine secondary metabolites. ${ }^{4}$ Notably, the 1,2-diaminoethyl group in the indole 3-position, in contrast to 2-aminoethyl, is uncharacteristic for terrestrial indole alkaloids.<smiles>[R]c1ccc2c(C(=O)C3=NCC(c4c[nH]c5cc([Tl])ccc45)N3)c[nH]c2c1</smiles>

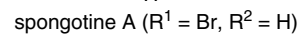
spongotine $B\left(R^{1}=H, R^{2}=B r\right)$ spongotine $C\left(R^{1}=R^{2}=B r\right)$

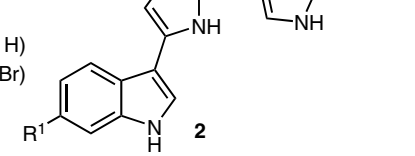

$$
\text { topsentin } A\left(R^{1}=R^{2}=H\right)
$$
bromodeoxytopsentin $\left(\mathrm{R}^{1}=\mathrm{Br}, \mathrm{R}^{2}=\mathrm{H}\right)$ isobromodeoxytopsentin $\left(\mathrm{R}^{1}=\mathrm{H}, \mathrm{R}^{2}=\mathrm{Br}\right)$<smiles>[R]c1ccc2c(C3=NCC(c4c[nH]c5cc([R])ccc45)NC3=O)c[nH]c2c1</smiles>

hamacanthin $A\left(R^{1}=R^{2}=B r\right)$ 6'-debromohamacanthin $A\left(R^{1}=B r, R^{2}=H\right)$ 6"-debromohamacanthin $A\left(R^{1}=H, R^{2}=B r\right)$<smiles>[R7]N1C[C@@H](c2c[nH]c3cc(Br)ccc23)N([C@@H](C)C=[In])C[C@@H]1c1c[nH]c2cc(Br)ccc12</smiles>

Figure 1 Several marine alkaloids containing an (indol-3-yl)ethanediamine fragment and their aromatized derivatives

Total syntheses of many of the natural products from this group have been reported; ${ }^{2,5,6}$ however, no synthesis of topsentin $\mathrm{C}$ has been reported. Compounds exhibiting anti- 
bacterial, cytotoxic, antiviral, and fungicidal properties were discovered among these alkaloids and their synthetic analogues. ${ }^{2,7}$

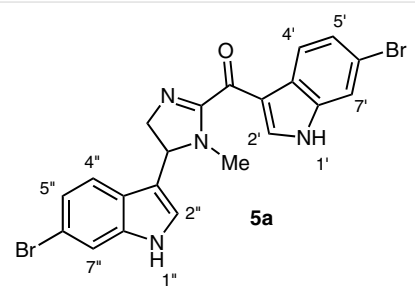

Figure 2 Proposed structure of topsentin C

(Indol-3-yl)ethane-1,2-diamines could be convenient synthetic precursors of topsentins, spongotines, and hamacanthins. ${ }^{5-c}$ Furthermore, these diamines are of independent interest because their simple derivatives were recently shown to be capable of preventing the development of resistance to fluoroquinolone antibiotics in Staphylococcus aureus. $^{8}$

Only two synthetic approaches to (indol-3-yl)ethane1,2-diamines have been published and neither of them allows the corresponding $N^{1}$-methyl derivatives to be produced..$^{5-c}$ It was also reported that diamines of this type with an unsubstituted indole $\mathrm{N}$ atom are relatively stable only as the salts. ${ }^{5 a}$ We propose a convenient preparative synthetic approach to (indol-3-yl)ethane-1,2-diamines (6) with a protected indole $\mathrm{N}$ atom that is based on mild reduction of 7, the addition product of $O$-pivaloylhydroxylamines (8) and 3-nitrovinylindoles (9) (Scheme 1). The proposed method has been used for the total synthesis of topsentin C, the previously proposed structure of which has been revised by us.

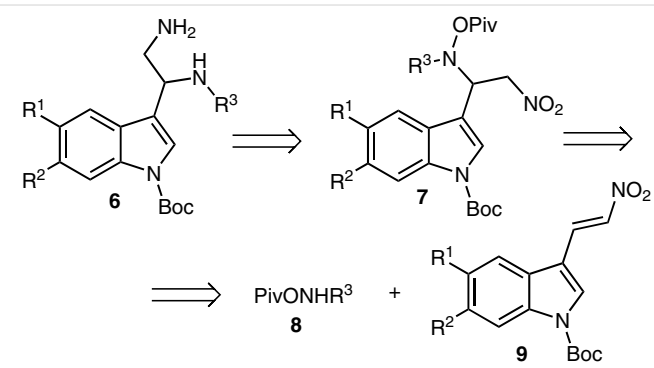

Scheme 1 Our Synthetic approach to (indol-3-yl)ethanediamine 6

Starting nitrovinylindoles $\mathbf{9}$, with tert-butoxycarbonylprotected indole $\mathrm{N}$ atoms, were synthesized from the corresponding indoles by formylation using $\mathrm{N}, \mathrm{N}$-dimethylformamide (DMF) and $\mathrm{SOCl}_{2}$ followed by condensation of the obtained aldehydes with nitromethane and addition of the protecting group in the presence of 4 - $(\mathrm{N}, \mathrm{N}$-dimethylamino)pyridine (DMAP) (Scheme 2). We also prepared 1-ace-

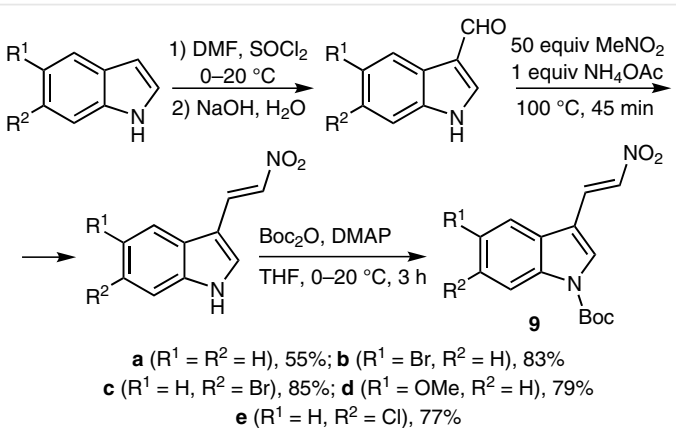

Scheme 2 Synthesis of 3-nitrovinylindoles 9; yields of three stages are shown

tyl-3-[(E)-2-nitrovinyl]-1H-indole (9f) and 1-methyl-3$[(E)$-2-nitrovinyl]-1H-indole $(\mathbf{9 g})$ according to described procedures. ${ }^{9,10}$

Reduction of the adducts of $\alpha, \beta$-unsaturated nitrocompounds with amines, $O$-alkylhydroxylamines or azide anion was proposed earlier for the synthesis of vicinal diamines. ${ }^{11-13}$ The first type of adduct was unstable, although they could be isolated as the more stable salts. ${ }^{11}$ Adducts with $O$-alkylhydroxylamines or azide anion were more stable. ${ }^{12,13}$ However, catalytic hydrogenation or heating with $\mathrm{Zn}$ dust in HOAc was required to cleave the hydroxylamine $\mathrm{N}-\mathrm{O}$ bond. ${ }^{12}$ Catalytic hydrogenation was also used to reduce the azido group. ${ }^{13}$ The proposed methods turned out to be ineffective for the synthesis of $\mathrm{Br}$-containing (indol-3yl)ethane-1,2-diamines $\mathbf{6}$. Thus, the products $\mathbf{1 0}$ from the reaction of methylamine or benzylamine with nitrovinylindole $\mathbf{9 b}$ could not be isolated; starting $\mathbf{9 b}$ was recovered and the reaction mixture formed a resin. Stable adduct $\mathbf{1 1}$

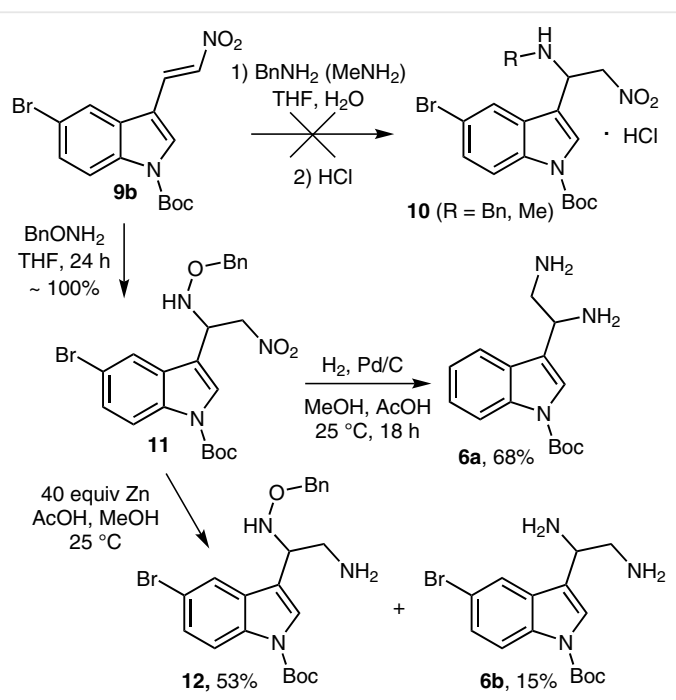

Scheme 3 Attempts to convert nitrovinylindole $9 b$ into the corresponding indolic diamines by using aliphatic amines or O-benzylhydroxylamine 
Table 1 Synthesis of Adducts 7

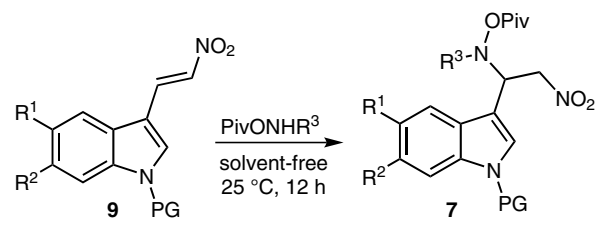

\begin{tabular}{cccccccc}
\hline Entry & Substrate & Product & $\mathrm{R}^{1}$ & $\mathrm{R}^{2}$ & $\mathrm{R}^{3}$ & $\mathrm{PG}$ & Yield (\%) \\
\hline 1 & $\mathbf{9 a}$ & $\mathbf{7 a}$ & $\mathrm{H}$ & $\mathrm{H}$ & $\mathrm{H}$ & Boc & 94 \\
2 & $\mathbf{9 b}$ & $\mathbf{7 b}$ & $\mathrm{Br}$ & $\mathrm{H}$ & $\mathrm{H}$ & $\mathrm{Boc}$ & 96 \\
3 & $\mathbf{9 a}$ & $\mathbf{7 c}$ & $\mathrm{H}$ & $\mathrm{H}$ & $\mathrm{Me}$ & $\mathrm{Boc}$ & 97 \\
4 & $\mathbf{9 b}$ & $\mathbf{7 d}$ & $\mathrm{Br}$ & $\mathrm{H}$ & $\mathrm{Me}$ & $\mathrm{Boc}$ & 89 \\
5 & $\mathbf{9 c}$ & $\mathbf{7 e}$ & $\mathrm{H}$ & $\mathrm{Br}$ & $\mathrm{Me}$ & Boc & 98 \\
6 & $\mathbf{9 d}$ & $\mathbf{7 f}$ & $\mathrm{OMe}$ & $\mathrm{H}$ & $\mathrm{Me}$ & Boc & 91 \\
7 & $\mathbf{9 e}$ & $\mathbf{7 g}$ & $\mathrm{H}$ & $\mathrm{Cl}$ & $\mathrm{Me}$ & Boc & 97 \\
8 & $\mathbf{9 f}$ & $\mathbf{7 h}$ & $\mathrm{H}$ & $\mathrm{H}$ & $\mathrm{Me}$ & $\mathrm{Ac}$ & 88 \\
9 & $\mathbf{9 g}$ & -[a] & $\mathrm{H}$ & $\mathrm{H}$ & $\mathrm{Me}$ & $\mathrm{Me}$ & 0 \\
\hline
\end{tabular}

${ }^{a}$ Nitrovinylindole $\mathbf{9 g}$, with a methyl on the indole $\mathrm{N}$ atom, did not react.

with $O$-benzylhydroxylamine was reduced as expected by $\mathrm{H}_{2}$ over $\mathrm{Pd} / \mathrm{C}$ with hydrogenolysis of the $\mathrm{C}-\mathrm{Br}$ bond to form diamine $\mathbf{6 a}$ (Scheme 3).

Indolic adduct $\mathbf{1 1}$ decomposed upon heating with $\mathrm{Zn}$ in $\mathrm{HOAc}$, and reduction with $\mathrm{Zn}$ under milder conditions was not complete. The yield of diamine $\mathbf{6 b}$ was $<15 \%$ even when the amount of $\mathrm{Zn}$ and the reaction time were increased; the main product was $\mathbf{1 2}$ (Scheme 3 ).

$\mathrm{O}$-Acylhydroxylamines have highly labile $\mathrm{N}-\mathrm{O}$ bonds and have recently been used in synthetic procedures based on sigmatropic shifts with cleavage of $\mathrm{N}-\mathrm{O}$ bonds ${ }^{14}$ in addition to amination reaction. ${ }^{15}$ Conjugate addition of $O$-acylhydroxylamines to electron-deficient alkenes has not yet been described, in contrast to $O$-alkylhydroxylamines. We decided to study the possibility of adding $O$-pivaloylhydroxylamine and its $N$-methyl derivative to nitrovinylindoles $\mathbf{9}$ followed by reduction of the resulting adducts. Derivatives of sterically hindered pivalic acid were chosen because they isomerize rather slowly into the corresponding hydroxamic acids, in contrast to the simpler $O$-acylhydroxylamines. ${ }^{16}$

Hydrochlorides of $\mathrm{O}$-pivaloylhydroxylamine and $\mathrm{N}$ methyl-O-pivaloylhydroxylamine were synthesized by using the previously reported methods. ${ }^{14 a, 16,17}$ The corresponding free bases were isolated immediately before performing the next step.

As it turned out, the reaction of nitrovinylindoles 9a with $\mathrm{O}$-pivaloylhydroxylamine (8a, 1.5 equiv) in $\mathrm{CH}_{2} \mathrm{Cl}_{2}$ was complete in 96 hours and gave target adduct $\mathbf{7 a}$. We also found that the solvent-free reaction was much faster. The reagents could be mixed and left overnight in a closed vessel. The nitrovinylindoles dissolved gradually, then crystals of the product formed. The solvent-free reaction was clearly advantageous from a green chemistry point of view. In this manner, we obtained the series of adducts $\mathbf{7 a}-\mathbf{h}$ in high yields (Table 1 ).

The next step was the reduction of adducts 7 . We decided to use $\mathrm{Zn}$ and acid, anticipating that their hydroxylamine $\mathrm{N}-\mathrm{O}$ bond would undergo reductive cleavage at room or reduced temperature. Thus, the reduction of $\mathbf{7 a}$ using $\mathrm{Zn}$ (10 equiv) and $\mathrm{HOAc}$ in $\mathrm{MeOH}$ afforded target diamine $\mathbf{6 a}$ in $34 \%$ yield (Table 2 , entry 1 ).

Table 2 Reduction of Adducts 7a,c

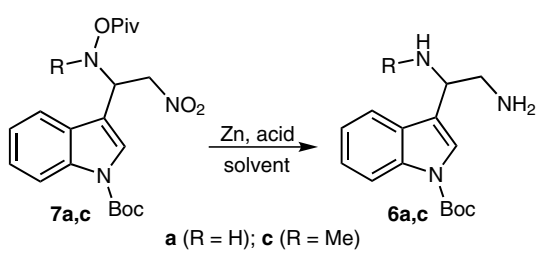

\begin{tabular}{|c|c|c|c|c|c|c|c|}
\hline Entry & $\mathrm{R}$ & Equiv. Zn & Acid (equiv) & Solvent & $t(\mathrm{~h})$ & $T\left({ }^{\circ} \mathrm{C}\right)$ & Yield (\%) \\
\hline 1 & $\mathrm{H}$ & 10 & $\mathrm{AcOH}(150)$ & $\mathrm{MeOH}$ & 2 & $0-20$ & 34 \\
\hline 2 & $\mathrm{H}$ & 10 & $\mathrm{AcOH}(150)$ & $\mathrm{MeOH}$ & 6 & $0-20$ & 31 \\
\hline 3 & $\mathrm{H}$ & 10 & $\mathrm{AcOH}(150)$ & $\mathrm{MeOH} / \mathrm{H}_{2} \mathrm{O} / \mathrm{EtOAC}$ & 2 & $0-20$ & 48 \\
\hline 4 & $\mathrm{H}$ & 20 & $\mathrm{AcOH}(150)$ & $\mathrm{MeOH} / \mathrm{H}_{2} \mathrm{O} / \mathrm{EtOAC}$ & 2 & $0-20$ & 57 \\
\hline 5 & $\mathrm{H}$ & 15 & $\mathrm{HCl}(30)$ & $\mathrm{MeOH} / \mathrm{EtOAC}$ & 2 & -10 to 5 & 65 \\
\hline 6 & $\mathrm{H}$ & 15 & $\mathrm{HBr}(30)$ & $\mathrm{MeOH} / \mathrm{EtOAc}$ & 2 & -10 to 5 & 84 \\
\hline 7 & $\mathrm{H}$ & 15 & $\mathrm{NH}_{4} \mathrm{Br}(15)$ & $\mathrm{EtOH} / \mathrm{H}_{2} \mathrm{O} / \mathrm{EtOAC}$ & 2 & 20 & 62 \\
\hline 8 & Me & 15 & $\mathrm{HBr}(30)$ & $\mathrm{MeOH} / \mathrm{EtOAC}$ & 2 & -10 to 5 & 68 \\
\hline 9 & $\mathrm{Me}$ & 20 & $\mathrm{HBr}(40)$ & $\mathrm{MeOH} / \mathrm{EtOAC}$ & 6 & -10 to 5 & 93 \\
\hline
\end{tabular}


The reaction proceeded rather quickly. However, it was accompanied by the formation of several unidentified side products. Increasing the reaction time did not lead to an increase in the yield of $\mathbf{6 a}$. We found that the yield could be increased by adding $\mathrm{H}_{2} \mathrm{O}$ and EtOAc to the reaction mixture (keeping the solution homogeneous) and by doubling the amount of $\mathrm{Zn}$ (cf. Table 2, entries 3 and 4). Replacing HOAc with concentrated $\mathrm{HCl}$ at reduced temperature led to a further increase in the yield (entry 5). Finally, the use of $\mathrm{HBr}$ (40\%) allowed target diamine $\mathbf{6 a}$ to be obtained with a very good yield (entry 6). It was also possible to conduct the reduction of adduct 7a in the presence of $\mathrm{NH}_{4} \mathrm{Br}$ (entry 7). The reduction of adduct $\mathbf{7 c}$, with a methyl group on the hydroxylamine $\mathrm{N}$ atom, was more difficult. However, increasing the reaction time and amount of $\mathrm{Zn}$ provided a high yield of diamine $\mathbf{6 c}$ (entry 9). The developed method was extended to adducts $\mathbf{7 b}$ and $\mathbf{7 d - f}$ (Table 3 ). Boc derivatives gave the corresponding diamines $\mathbf{6 b}$ and $\mathbf{6 d - g}$ in good and high yields, whereas diamine $\mathbf{6 h}$, with an acetyl on the indole $\mathrm{N}$ atom, was unstable and decomposed even in solution.

Table 3 Synthesis of Diamines 6

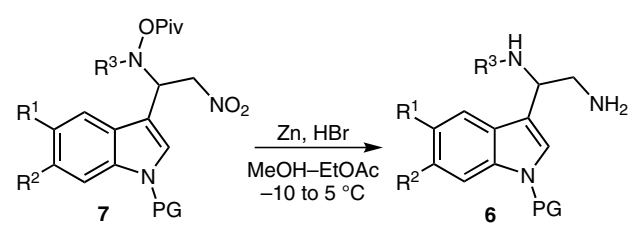

\begin{tabular}{cccccccc}
\hline Entry & Substrate & Product & $\mathrm{R}^{1}$ & $\mathrm{R}^{2}$ & $\mathrm{R}^{3}$ & $\mathrm{PG}$ & Yield (\%) \\
\hline $1^{\mathrm{a}}$ & $\mathbf{7 a}$ & $\mathbf{6 a}$ & $\mathrm{H}$ & $\mathrm{H}$ & $\mathrm{H}$ & Boc & 84 \\
$2^{\mathrm{a}}$ & $\mathbf{7 b}$ & $\mathbf{6 b}$ & $\mathrm{Br}$ & $\mathrm{H}$ & $\mathrm{H}$ & $\mathrm{Boc}$ & 95 \\
$3^{\mathrm{b}}$ & $\mathbf{7 c}$ & $\mathbf{6 c}$ & $\mathrm{H}$ & $\mathrm{H}$ & $\mathrm{Me}$ & Boc & 93 \\
$4^{\mathrm{b}}$ & $\mathbf{7 d}$ & $\mathbf{6 d}$ & $\mathrm{Br}$ & $\mathrm{H}$ & $\mathrm{Me}$ & Boc & 86 \\
$5^{\mathrm{b}}$ & $\mathbf{7 e}$ & $\mathbf{6 e}$ & $\mathrm{H}$ & $\mathrm{Br}$ & $\mathrm{Me}$ & Boc & 92 \\
$6^{\mathrm{b}}$ & $\mathbf{7 f}$ & $\mathbf{6 f}$ & $\mathrm{OMe}$ & $\mathrm{H}$ & $\mathrm{Me}$ & Boc & 75 \\
$7^{\mathrm{b}}$ & $\mathbf{7 g}$ & $\mathbf{6 g}$ & $\mathrm{H}$ & $\mathrm{Cl}$ & $\mathrm{Me}$ & Boc & 90 \\
$8^{\mathrm{b}}$ & $\mathbf{7 h}$ & $\mathbf{6 h}$ & $\mathrm{H}$ & $\mathrm{H}$ & $\mathrm{Me}$ & Ac & $-^{\mathrm{c}}$ \\
\hline
\end{tabular}

a Reaction conditions: $\mathrm{Zn}$ (15 equiv), $\mathrm{HBr}$ (30 equiv), $2 \mathrm{~h}$.

b Reaction conditions: $\mathrm{Zn}$ (20 equiv), $\mathrm{HBr}$ (40 equiv), $6 \mathrm{~h}$.

' Decomposition of the product occurred.

Having established a convenient preparative method for $N^{1}$-methyl(indol-3-yl)ethane-1,2-diamines, we focused on the total synthesis of the proposed structure for topsentin C (5a), which is related to spongotines $\mathbf{1}$ and other previously isolated topsentines. The imidazoline fragment of $\mathbf{5 a}$ and its analogue $\mathbf{5 b}$, without a $\mathrm{Br}$ atom, was constructed by using the previously reported synthetic method for imidazolines that involved condensation of the vicinal diamines with aldehydes (including $\alpha$-keto aldehydes) followed by oxidation of the resulting cyclic aminal. ${ }^{5 c, 18}$ The required indolylglyox- als 14a and 14b were prepared from corresponding 3acetylindoles 15a and 15b through iodination followed by Kornblum oxidation (Scheme 4). ${ }^{19}$

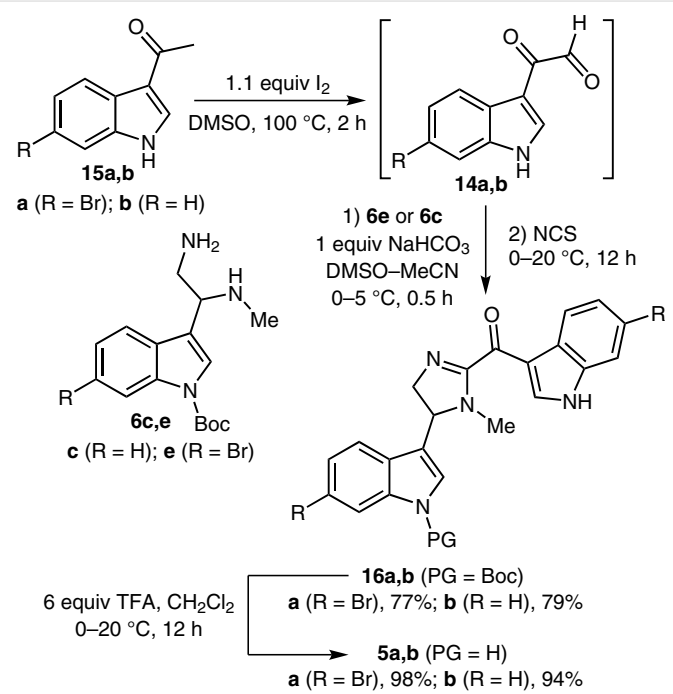

Scheme 4 Synthesis of spongotine analogues $\mathbf{5 a}$ and $\mathbf{5 b}$

The aforementioned syntheses of indolylglyoxals $\mathbf{1 4 a}$ and $\mathbf{1 4 b}$, condensations with diamines $\mathbf{6 c}$ and $\mathbf{6 e}$, and subsequent oxidations to imidazolines $\mathbf{1 6 a}$ and $\mathbf{1 6 b}$ were carried out in one pot. This made the developed procedure attractive for preparative reactions. The protecting group could be removed to afford $\mathbf{5 a}$ and $\mathbf{5 b}$. As it turned out, the spectral characteristics of $\mathbf{5 a}$ synthesized by us and the characteristics of topsentin $C$ that was isolated from the natural source, differed dramatically. Therefore, the initially proposed structure of topsentin $C$ had to be revised. Thus, the synthesized 5a was a methylated spongotine $C$ derivative that has not yet been observed in nature. The developed method enables analogues of spongotines and topsentins to be synthesized to study their biological properties, which are known to change abruptly if even a single methyl is added to the molecule. ${ }^{17}$

We assumed that natural topsentin C was structurally related to hamacanthins A (3) but not spongotines (1), and was the 1-methyl derivative of hamacanthin A 17a (Figure $3),{ }^{20}$ which should have a set of NMR signals similar to that of $5 \mathbf{a}$.

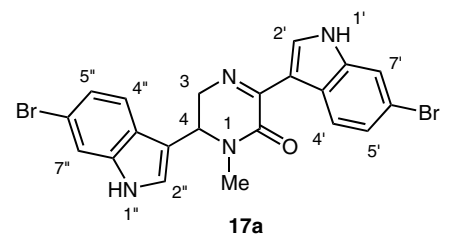

Figure 3 Revised structure of topsentin C 
<smiles>[Y6]NC(CN)c1cn(C(Br)(Br)Br)c2cc([R])ccc12</smiles>

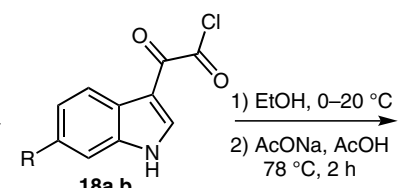

$\mathbf{a}(\mathrm{R}=\mathrm{Br}) ; \mathbf{b}(\mathrm{R}=\mathrm{H})$

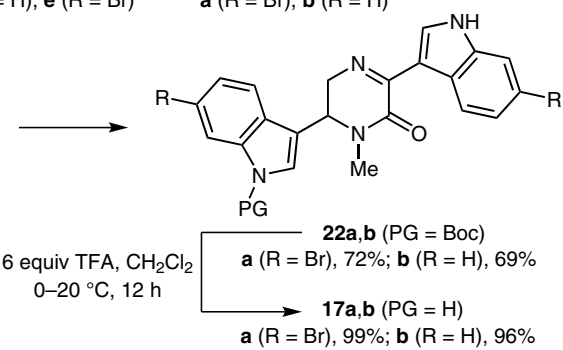

Scheme 5 Synthesis of topsentin $C$ and its analogue

We synthesized bis(indolyl)dihydropyrazinones 17a and $\mathbf{1 7 b}$ through cyclization of diamines $\mathbf{6 e}$ and $\mathbf{6 c}$ with indoleglyoxylic acid chlorides $\mathbf{1 8 a}$ and $\mathbf{1 8 b}$ to confirm this hypothesis (Scheme 5).

Acid chlorides $\mathbf{1 8 a}$ and $\mathbf{1 8 b}$ were obtained through acylation of the corresponding indoles by using oxalylchloride according to published methods. ${ }^{6 \mathrm{k}}$ The reaction first gave a mixture of amides $\mathbf{1 9}$ and 20, which were further cyclized without isolation (Scheme 6). As noted earlier during the development of synthetic methods for hamacanthins, these amides can undergo reversible transformations under the cyclization conditions via intermediate $\mathbf{2 1}$, and can form a

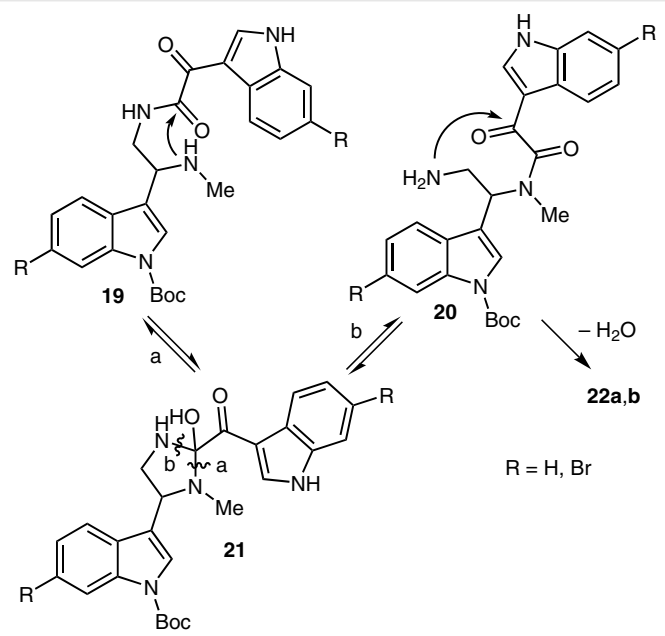

Scheme 6 Mechanism of dihydropyrazinone ring formation

mixture of isomeric bis(indolyl)dihydropyrazinones. ${ }^{6 b}$ In this case, the cyclization was unidirectional because of the methyl group, so that 22a and $\mathbf{2 2 b}$ were isolated only. ${ }^{21}$ These compounds were converted into target $\mathbf{1 7} \mathbf{a}$ and $\mathbf{1 7} \mathbf{b}$ by removing the protecting group. The structure of compound 17a was established by X-ray crystallographic analysis (Figure 4).

The spectral characteristics of bis(indolyl)dihydropyrazinone 17a were consistent with those of natural topsentin $\mathrm{C}^{3,22}$ in contrast to imidazoline $\mathbf{5 a}$ (Table 4 ). This data con-

Table $4{ }^{1} \mathrm{H}$ NMR Data for Natural Topsentin C and for Compounds $\mathbf{1 7 a}$ and $\mathbf{5} \mathbf{a}^{\mathrm{a}}$

\begin{tabular}{|c|c|c|c|}
\hline${ }^{1} \mathrm{H}$ & $17 a$ & Topsentin $C^{3}$ & $5 a$ \\
\hline $\mathrm{N}(1) \mathrm{CH}_{3}$ & $3.04(s)$ & $3.05(\mathrm{~s})$ & $2.82(\mathrm{~s})$ \\
\hline $\mathrm{CHCH}_{a} \mathrm{H}_{\mathrm{b}}$ & $4.26(\mathrm{dd}, J=16.5,5.5)$ & $4.27(\mathrm{dd}, J=16.5,5.3)$ & $3.91(\mathrm{dd}, J=15.3,10.3)$ \\
\hline $\mathrm{CHCH}_{\mathrm{a}} \mathrm{H}_{\mathrm{b}}$ & $4.41(\mathrm{dd}, J=16.5,5.2)$ & $4.41(\mathrm{dd}, J=16.5,5.2)$ & $4.31(\mathrm{dd}, J=15.3,11.3)$ \\
\hline $\mathrm{CHCH}_{\mathrm{a}} \mathrm{H}_{\mathrm{b}}$ & $5.15(\mathrm{dd}, J=5.5,5.2)$ & $5.16(\mathrm{ddd}, J=5.3,5.2,<1)$ & $4.89(\mathrm{dd}, J=11.3,10.3)$ \\
\hline \multicolumn{4}{|l|}{ Indolic H } \\
\hline $1^{\prime}$ & $10.70(\mathrm{br} \mathrm{s})$ & $10.71(\mathrm{br} \mathrm{s})$ & 11.37 (br s) \\
\hline $2^{\prime}$ & $8.62(\mathrm{~d}, J=2.75)$ & $8.62(\mathrm{~d}, J=2.7)$ & $8.68(\mathrm{~s})$ \\
\hline $4^{\prime}$ & $8.37(\mathrm{~d}, J=8.6)$ & $8.37(\mathrm{~d}, J=8.7)$ & $8.32(\mathrm{~d}, J=8.5)$ \\
\hline $5^{\prime}$ & $7.17-7.23(\mathrm{~m})^{\mathrm{b}}$ & $7.20(\mathrm{dd}, J=8.7,1.8)$ & $7.41(\mathrm{dd}, J=8.5,1.7)$ \\
\hline $7^{\prime}$ & $7.66(\mathrm{~d}, J=1.8)$ & $7.66(\mathrm{~d}, J=1.8)$ & $7.76(\mathrm{~d}, J=1.6)$ \\
\hline $1^{\prime \prime}$ & 10.34 (br s) & 10.34 (br s) & 10.42 (br s) \\
\hline $2^{\prime \prime}$ & $7.17-7.23(\mathrm{~m})^{\mathrm{b}}$ & $7.22(\mathrm{dd}, J=2.5,<1)$ & $7.45(\mathrm{~d}, J=2.1)$ \\
\hline $4^{\prime \prime}$ & $7.69(\mathrm{~d}, J=8.6)$ & $7.69(\mathrm{~d}, J=8.5)$ & $7.63(\mathrm{~d}, J=8.5)$ \\
\hline $5^{\prime \prime}$ & $7.17-7.23(\mathrm{~m})^{\mathrm{b}}$ & $7.20(\mathrm{dd}, J=8.5,1.7)$ & $7.17(\mathrm{dd}, J=8.5,1.7)$ \\
\hline $7^{\prime \prime}$ & $7.63(\mathrm{~d}, J=1.7)$ & $7.63(\mathrm{~d}, J=1.7)$ & $7.65(d, J=1.6)$ \\
\hline
\end{tabular}

a Recorded in acetone- $d_{6}$; shift in ppm, coupling in $\mathrm{Hz}$.

${ }^{\mathrm{b}}$ Double resonance experiments and COSY gave the same interpretation of these signals as shown for natural topsentin C (see the Supporting Information). 


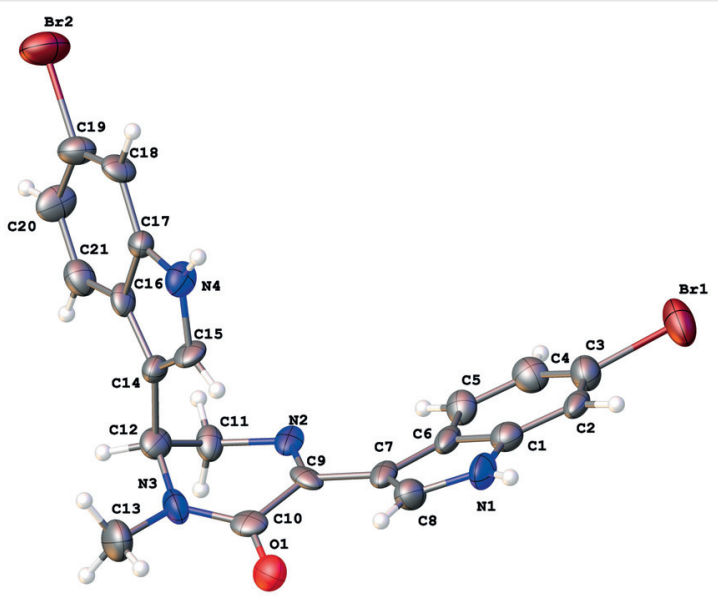

Figure 4 Molecular structure of 17a presented as ADP ellipsoids at $50 \%$ probability

firmed our hypothesis regarding the structure of the natural topsentin C.

Thus, we have developed a convenient preparative synthetic method to prepare (indol-3-yl)ethane-1,2-diamines and found that the natural topsentin $C$ has the structure 17a. The total synthesis of racemic 17a was carried out in seven steps from 6-bromoindole $\mathbf{9 c}$ in $55 \%$ overall yield.

Starting reagents were either purchased from commercial sources and used without additional purification or were prepared according to reported procedures. ${ }^{1} \mathrm{H}$ and ${ }^{13} \mathrm{C}$ NMR spectra were acquired with 400,500 , or $600 \mathrm{MHz}$ spectrometers at r.t. and referenced to the residual signals of the solvent (for ${ }^{1} \mathrm{H}$ and ${ }^{13} \mathrm{C}$ ). The solvents for NMR samples were DMSO- $d_{6}, \mathrm{CDCl}_{3}$, and acetone- $d_{6}$. Chemical shifts are reported in parts per million $(\delta, \mathrm{ppm})$. Coupling constants are reported in Hertz $(J, H z)$. The peak patterns are indicated as: s, singlet; d, doublet; t, triplet; q, quadruplet; m, multiplet; dd, doublet of doublets; br s, broad singlet. Signal assignment was based on COSY, HSQC, HMBC and NOESY experiments. Infrared spectra were measured with an Infralum FT-801 FT/IR instrument. The wavelengths are reported in reciprocal centimeters $\left(\mathrm{v}_{\max }, \mathrm{cm}^{-1}\right)$. Mass spectra were recorded with LCMS-8040 triple quadrupole liquid chromatograph mass-spectrometer from Shimadzu (ESI) and Kratos MS-30 mass spectrometer (EI, $70 \mathrm{eV}$ ). Elemental analysis was performed with an Euro Vector EA-3000 elemental analyzer. The X-ray data collection of 17a was performed with a Bruker APEX-II CCD diffractometer at $120 \mathrm{~K}$. Details of the X-ray structure determination are given in the Supporting Information. The progress of the reaction was monitored by TLC and the spots were visualized under UV light (254 or $365 \mathrm{~nm}$ ). Column chromatography was performed using silica gel (230-400 mesh). Melting points were determined with a SMP-10 apparatus and are uncorrected. Solvents were distilled and dried according to standard procedures.

tert-Butyl 3-[(E)-2-Nitrovinyl]-1H-indole-1-carboxylate (9a)23 $\mathrm{SOCl}_{2}(13.7 \mathrm{~mL}, 0.188 \mathrm{~mol})$ at $0-5{ }^{\circ} \mathrm{C}$ was added dropwise to anhydrous DMF ( $14.6 \mathrm{~mL}, 0.188 \mathrm{~mol}$ ) and the solution was stirred at r.t. for $30 \mathrm{~min}$. The resulting mixture was degassed under vacuum and the residue was diluted with anhydrous DMF (26 mL) and treated with a solution of indole $(20.0 \mathrm{~g}, 0.171 \mathrm{~mol})$ in DMF $(10 \mathrm{~mL})$ dropwise at 0$5{ }^{\circ} \mathrm{C}$ over $10 \mathrm{~min}$. The mixture was stirred at r.t. for $40 \mathrm{~min}$, then treated with ice ( $100 \mathrm{~g})$ and $\mathrm{NaOH}$ solution $(30 \%)$ was added until $\mathrm{pH}$ ca. 8 . The mixture was heated to reflux, and cooled. The resulting precipitate was filtered off, rinsed with $\mathrm{H}_{2} \mathrm{O}(5 \times)$, dried in air, heated at reflux in EtOH (40 mL), cooled, filtered off again, and dried in air to afford $1 H$-indole-3-carbaldehyde (22.5 g, 91\%).

A solution of $1 \mathrm{H}$-indole-3-carbaldehyde $(12.5 \mathrm{~g}, 0.086 \mathrm{~mol})$ and $\mathrm{NH}_{4} \mathrm{OAc}$ ( $6.6 \mathrm{~g}, 0.086 \mathrm{mmol}$ ) in $\mathrm{CH}_{3} \mathrm{NO}_{2}$ (46 mL, $0.861 \mathrm{~mol}$ ) was heated at reflux for $45 \mathrm{~min}$, cooled to r.t., and diluted with $\mathrm{H}_{2} \mathrm{O}(150 \mathrm{~mL})$. The resulting precipitate was filtered off, washed with $\mathrm{H}_{2} \mathrm{O}(5 \times)$, dried on air, heated at reflux in EtOH (60 mL), cooled, filtered off again and dried on air to afford 3-[(E)-2-nitrovinyl]-1H-indole (11.3 g, 70\%).

To a solution of 3-[(E)-2-nitrovinyl]-1H-indole (5.6 g, $30 \mathrm{mmol})$ and DMAP $(0.37 \mathrm{~g}, 3.0 \mathrm{mmol})$ in anhydrous THF $(30 \mathrm{~mL})$ was added dropwise a solution of $\mathrm{Boc}_{2} \mathrm{O}(9.9 \mathrm{~g}, 45.4 \mathrm{mmol})$ in anhydrous THF $(30 \mathrm{~mL})$ at $0-5{ }^{\circ} \mathrm{C}$ over a period of $15 \mathrm{~min}$. The reaction mixture was stirred at r.t. for $3 \mathrm{~h}$, and concentrated in vacuo. The residue was dissolved in $\mathrm{CH}_{2} \mathrm{Cl}_{2}(100 \mathrm{~mL})$, washed with citric acid solution $(10 \%, 30 \mathrm{~mL}), \mathrm{H}_{2} \mathrm{O}$ $(30 \mathrm{~mL})$, and concd $\mathrm{NaCl}$ solution $(20 \mathrm{~mL})$, then dried over anhydrous $\mathrm{Na}_{2} \mathrm{SO}_{4}$. The $\mathrm{CH}_{2} \mathrm{Cl}_{2}$ was evaporated in vacuo and the residue was heated at reflux in $\mathrm{MeOH}(30 \mathrm{~mL})$ and cooled. The resulting precipitate was filtered off, washed twice with cold hexane, and dried on air to afford tert-butyl 3-[(E)-2-nitrovinyl]-1H-indole-1-carboxylate.

Yield: $7.6 \mathrm{~g}$ (55\% from indole); yellow solid; $\mathrm{mp} 144-145{ }^{\circ} \mathrm{C}(\mathrm{MeOH})$. ${ }^{1} \mathrm{H}$ NMR $\left(600 \mathrm{MHz}\right.$, DMSO- $\left.d_{6}\right): \delta=8.55(\mathrm{~s}, 1 \mathrm{H}), 8.36(\mathrm{~d}, J=13.7 \mathrm{~Hz}$, $\left.1 \mathrm{H}, \mathrm{CH}=\mathrm{CHNO}_{2}\right), 8.19\left(\mathrm{~d}, J=13.7 \mathrm{~Hz}, 1 \mathrm{H}, \mathrm{CH}=\mathrm{CHNO}_{2}\right), 8.11$ (d, $J=$ $8.1 \mathrm{~Hz}, 1 \mathrm{H}), 8.02(\mathrm{~d}, J=7.1 \mathrm{~Hz}, 1 \mathrm{H}), 7.40-7.46(\mathrm{~m}, 1 \mathrm{H}), 7.33-7.39(\mathrm{~m}$, $1 \mathrm{H}), 1.64\left(\mathrm{~s}, 9 \mathrm{H}, \mathrm{C}\left(\mathrm{CH}_{3}\right)_{3}\right)$.

${ }^{13} \mathrm{C}$ NMR (150 MHz, DMSO- $\left.d_{6}\right): \delta=148.79,136.50,136.06,134.27$, 132.37, 127.15, 126.14, 124.54, 121.40, 115.67, 112.76, 85.72, 28.08 (3C).

tert-Butyl 5-Bromo-3-[(E)-2-nitrovinyl]-1H-indole-1-carboxylate (9b); Typical Procedure

5-Bromoindole (1.35 g, $6.9 \mathrm{mmol}$ ) was formylated as described for $1 \mathrm{H}$-indole-3-carbaldehyde to produce 6 -bromo- $1 \mathrm{H}$-indole-3-carbaldehyde (1.50 g, 97\%) with the exception that the obtained product was not purified by refluxing in EtOH.

5-Bromo- $1 \mathrm{H}$-indole-3-carbaldehyde ( $1.50 \mathrm{~g}, 6.7 \mathrm{mmol}$ ) and $\mathrm{NH}_{4} \mathrm{OAc}$ $(0.52 \mathrm{~g}, 6.7 \mathrm{mmol})$ were heated at reflux in $\mathrm{CH}_{3} \mathrm{NO}_{2}(18 \mathrm{~mL}, 335$ $\mathrm{mmol}$ ) for $45 \mathrm{~min}$, cooled to r.t., and treated with $\mathrm{H}_{2} \mathrm{O}(70 \mathrm{~mL})$. The product was extracted with EtOAc $(70 \mathrm{~mL})$, washed with $\mathrm{H}_{2} \mathrm{O}(5 \times 50$ $\mathrm{mL}$ ) and $\mathrm{NaCl}$ solution $(20 \mathrm{~mL})$, and dried over anhydrous $\mathrm{Na}_{2} \mathrm{SO}_{4}$. The solvent was removed in vacuo to afford 6-bromo-3-[(E)-2-nitrovinyl]$1 H$-indole ( $1.72 \mathrm{~g}, 96 \%$, brownish crystals), which was dried in vacuo and used without further purification.

A suspension of 5-bromo-3-[(E)-2-nitrovinyl]-1H-indole (1.72 g, 6.4 $\mathrm{mmol})$ and DMAP $(0.08 \mathrm{~g}, 0.64 \mathrm{mmol})$ in anhydrous THF $(7 \mathrm{~mL})$ was treated with a solution of $\mathrm{Boc}_{2} \mathrm{O}(2.1 \mathrm{~g}, 9.7 \mathrm{mmol})$ in anhydrous THF $(7 \mathrm{~mL})$ dropwise at $0-5{ }^{\circ} \mathrm{C}$ over a period of $15 \mathrm{~min}$, stirred at r.t. for 3 $\mathrm{h}$, and concentrated in vacuo. The residue was dissolved in $\mathrm{CH}_{2} \mathrm{Cl}_{2}(50$ $\mathrm{mL})$, washed with citric acid solution $(10 \%, 20 \mathrm{~mL}), \mathrm{H}_{2} \mathrm{O}(20 \mathrm{~mL})$, and concd $\mathrm{NaCl}$ solution $(15 \mathrm{~mL})$, and dried over anhydrous $\mathrm{Na}_{2} \mathrm{SO}_{4}$. The $\mathrm{CH}_{2} \mathrm{Cl}_{2}$ was evaporated in vacuo and the residue was purified by chromatography on a column of silica gel (EtOAc-hexane, 6:1) to provide tert-butyl 5-bromo-3-[(E)-2-nitrovinyl]-1H-indole-1-carboxylate.

Yield: $2.11 \mathrm{~g}$ (83\% from 5-bromoindole); pale-yellow solid; mp 156$157^{\circ} \mathrm{C}(\mathrm{MeOH}) ; R_{f}=0.55$ (EtOAc-hexane, $1: 8$ ). 
IR (film): 3130, 2983, 2300 w, 1741 s (CO), 1639, 1509, 1452, 1368, $1344,1240,1153,1107,956,853,803,764,649,613,579 \mathrm{~cm}^{-1}$.

${ }^{1} \mathrm{H}$ NMR (400 MHz, DMSO- $d_{6}$ ): $\delta=8.57(\mathrm{~s}, 1 \mathrm{H}), 8.33(\mathrm{~d}, J=13.7 \mathrm{~Hz}$, $\left.1 \mathrm{H}, \mathrm{CH}=\mathrm{CHNO}_{2}\right), 8.27(\mathrm{~d}, J=1.9 \mathrm{~Hz}, 1 \mathrm{H}), 8.26(\mathrm{~d}, J=13.7 \mathrm{~Hz}, 1 \mathrm{H}$, $\left.\mathrm{CH}=\mathrm{CHNO}_{2}\right), 8.02(\mathrm{~d}, J=8.8 \mathrm{~Hz}, 1 \mathrm{H}), 7.56(\mathrm{dd}, J=8.8,1.9 \mathrm{~Hz}, 1 \mathrm{H}), 1.65$ $\left(\mathrm{s}, 9 \mathrm{H}, \mathrm{C}\left(\mathrm{CH}_{3}\right)_{3}\right)$.

${ }^{13} \mathrm{C}$ NMR $\left(100 \mathrm{MHz}\right.$, DMSO- $\left.d_{6}\right): \delta=147.89,136.57,134.27,133.94$, $130.89,128.52,128.16,123.08,116.83,116.76,111.47,85.53,27.48$ (3C).

MS (EI, $70 \mathrm{eV}): m / z(\%)=368 / 366(4)[\mathrm{M}]^{+}, 312 / 310(7)\left[\mathrm{M}-\mathrm{C}_{4} \mathrm{H}_{8}\right]^{+}$, 268/266 (31) [M - $\left.\mathrm{C}_{4} \mathrm{H}_{8}-\mathrm{CO}_{2}\right]^{+}, 219$ (21), 140 (30), 57 (100).

Anal. Calcd for $\mathrm{C}_{15} \mathrm{H}_{15} \mathrm{BrN}_{2} \mathrm{O}_{4}$ : C, 49.06; H, 4.12; N, 7.63. Found: C, 49.19; H, 4.15; N, 7.55.

\section{tert-Butyl 6-Bromo-3-[(E)-2-nitrovinyl]-1H-indole-1-carboxylate (9c)}

Yield: $2.15 \mathrm{~g}$ (85\% from 6-bromoindole); pale-yellow solid; mp 124$126^{\circ} \mathrm{C}\left(\mathrm{MeOH} ; R_{f}=0.62\right.$ (EtOAc-hexane, $\left.1: 8\right)$.

IR (film): 3129, 2985, $2300 \mathrm{w}, 1740 \mathrm{~s}$ (CO), 1635, 1505, 1427, 1341, 1299, 1238, 1144, 1106, 965, 842, 808, 764, 726, 650, $591 \mathrm{~cm}^{-1}$.

${ }^{1} \mathrm{H} \mathrm{NMR}\left(600 \mathrm{MHz}, \mathrm{CDCl}_{3}\right): \delta=8.43(\mathrm{~s}, 1 \mathrm{H}), 8.12(\mathrm{~d}, J=13.6 \mathrm{~Hz}, 1 \mathrm{H}$, $\left.\mathrm{CH}=\mathrm{CHNO}_{2}\right), 7.98(\mathrm{~s}, 1 \mathrm{H}), 7.71\left(\mathrm{~d}, J=13.6 \mathrm{~Hz}, 1 \mathrm{H}, \mathrm{CH}=\mathrm{CHNO}_{2}\right), 7.54$ $(\mathrm{d}, J=8.3 \mathrm{~Hz}, 1 \mathrm{H}), 7.48(\mathrm{dd}, J=8.3,1.7 \mathrm{~Hz}, 1 \mathrm{H}), 1.71\left(\mathrm{~s}, 9 \mathrm{H}, \mathrm{C}\left(\mathrm{CH}_{3}\right)_{3}\right)$. ${ }^{13} \mathrm{C}$ NMR $\left(150 \mathrm{MHz}, \mathrm{CDCl}_{3}\right): \delta=148.29,137.09,136.22,132.14$, 130.97, 127.58, 125.79, 121.20, 119.87, 119.27, 112.37, 86.27, 28.15 (3C).

MS (EI, $70 \mathrm{eV}): m / z(\%)=368 / 366(7)[\mathrm{M}]^{+}, 312 / 310(11)\left[\mathrm{M}-\mathrm{C}_{4} \mathrm{H}_{8}\right]^{+}$, 268/266 (40) [M - $\left.\mathrm{C}_{4} \mathrm{H}_{8}-\mathrm{CO}_{2}\right]^{+}, 221$ (18), 140 (21), 57 (100).

Anal. Calcd for $\mathrm{C}_{15} \mathrm{H}_{15} \mathrm{BrN}_{2} \mathrm{O}_{4}$ : C, 49.06; $\mathrm{H}, 4.12 ; \mathrm{N}, 7.63$. Found: C, 49.24; H, 4.19; N, 7.60.

\section{tert-Butyl 5-Methoxy-3-[(E)-2-nitrovinyl]-1H-indole-1-carboxyl- ate (9d)}

Yield: $1.73 \mathrm{~g}$ (79\% from 5-methoxyindole); yellow solid; mp 153$154{ }^{\circ} \mathrm{C}(\mathrm{MeOH}) ; R_{f}=0.52$ (EtOAc-hexane, $1: 8$ ).

IR (film): 3139, 2982, 2945, 2296 w, 1732 s (CO), 1633, 1544, 1500, $1374,1325,1251,1161,1097,1024,969,834,800,765,640,592 \mathrm{~cm}^{-}$ 1 .

${ }^{1} \mathrm{H}$ NMR (600 MHz, DMSO- $\left.d_{6}\right): \delta=8.52(\mathrm{~s}, 1 \mathrm{H}), 8.37(\mathrm{~d}, J=13.6 \mathrm{~Hz}$, $\left.1 \mathrm{H}, \mathrm{CH}=\mathrm{CHNO}_{2}\right), 8.24\left(\mathrm{~d}, J=13.6 \mathrm{~Hz}, 1 \mathrm{H}, \mathrm{CH}=\mathrm{CHNO}_{2}\right), 7.98(\mathrm{~d}, J=$ $9.0 \mathrm{~Hz}, 1 \mathrm{H}), 7.45(\mathrm{~d}, J=2.6 \mathrm{~Hz}, 1 \mathrm{H}), 7.01(\mathrm{dd}, J=9.0,2.6 \mathrm{~Hz}, 1 \mathrm{H}), 3.86$ (s, $\left.3 \mathrm{H}, \mathrm{OCH}_{3}\right), 1.64\left(\mathrm{~s}, 9 \mathrm{H}, \mathrm{C}\left(\mathrm{CH}_{3}\right)_{3}\right)$.

${ }^{13} \mathrm{C}$ NMR (150 MHz, DMSO- $\left.d_{6}\right): \delta=156.54,148.24,135.93,133.46$, 131.80, 129.90, 127.84, 115.87, 114.40, 112.13, 103.33, 85.04, 55.73, $27.56(3 \mathrm{C})$.

MS (EI, $70 \mathrm{eV}): m / z(\%)=319(8), 318(41)[\mathrm{M}]^{+}, 263(17), 262(81)[\mathrm{M}$ $\left.-\mathrm{C}_{4} \mathrm{H}_{8}\right]^{+}, 219(23), 218(28)\left[\mathrm{M}-\mathrm{C}_{4} \mathrm{H}_{8}-\mathrm{CO}_{2}\right]^{+}, 186(31), 175$ (69), 156 (29), 57 (100).

Anal. Calcd for $\mathrm{C}_{16} \mathrm{H}_{18} \mathrm{~N}_{2} \mathrm{O}_{5}$ : C, 60.37; H, 5.70; N, 8.80. Found: C, 60.28; H, 5.76; N, 8.79.

\section{tert-Butyl 6-Chloro-3-[(E)-2-nitrovinyl]-1H-indole-1-carboxylate} (9e)

Yield: $1.71 \mathrm{~g}$ (77\% from 6-chloroindole); $\mathrm{mp} 130-132{ }^{\circ} \mathrm{C}(\mathrm{MeOH}) ; R_{f}=$ 0.62 (EtOAc-hexane, 1:8).
IR (film): 3131, 2983, 2294 w, 1741 s (CO), 1634, 1508, 1431, 1340, $1299,1240,1145,1103,968,845,810,762,732,660,599 \mathrm{~cm}^{-1}$.

${ }^{1} \mathrm{H}$ NMR (400 MHz, DMSO- $\left.d_{6}\right): \delta=8.54(\mathrm{~s}, 1 \mathrm{H}), 8.32(\mathrm{~d}, J=13.7 \mathrm{~Hz}$, $\left.1 \mathrm{H}, \mathrm{CH}=\mathrm{CHNO}_{2}\right), 8.17\left(\mathrm{~d}, J=13.7 \mathrm{~Hz}, 1 \mathrm{H}, \mathrm{CH}=\mathrm{CHNO}_{2}\right), 8.08(\mathrm{~d}, J=$ $1.7 \mathrm{~Hz}, 1 \mathrm{H}), 8.04(\mathrm{~d}, J=8.6 \mathrm{~Hz}, 1 \mathrm{H}), 7.36(\mathrm{dd}, J=8.6,1.7 \mathrm{~Hz}, 1 \mathrm{H}), 1.65$ (s, $\left.9 \mathrm{H}, \mathrm{C}\left(\mathrm{CH}_{3}\right)_{3}\right)$.

${ }^{13} \mathrm{C}$ NMR (100 MHz, DMSO- $\left.d_{6}\right): \delta=147.85,136.40,135.86,133.93$, $131.10,130.14,125.40,123.99,122.14,114.88,111.96,85.68,27.45$ (3C).

MS (EI, $70 \mathrm{eV}): m / z(\%)=324(14) / 322(42)[\mathrm{M}]^{+}, 268(9) / 266(27)[\mathrm{M}-$ $\left.\mathrm{C}_{4} \mathrm{H}_{8}\right]^{+}, 224(16) / 222(55)\left[\mathrm{M}-\mathrm{C}_{4} \mathrm{H}_{8}-\mathrm{CO}_{2}\right]^{+}, 179(26), 174(26), 113$ (97), 57 (100).

Anal. Calcd for $\mathrm{C}_{15} \mathrm{H}_{15} \mathrm{ClN}_{2} \mathrm{O}_{4}$ : C, 55.82; $\mathrm{H}, 4.68 ; \mathrm{N}, 8.68$. Found: C, 55.70; H, 4.73; N, 8.63.

\section{tert-Butyl 3-\{1-[(Benzyloxy)amino]-2-nitroethyl\}-5-bromo-1H- indole-1-carboxylate (11)}

To a solution of 5-bromo-3-[(E)-2-nitrovinyl]-1H-indole (0.55 g, 1.5 $\mathrm{mmol}$ ) in anhydrous THF ( $3 \mathrm{~mL}), O$-benzylhydroxylamine $(0.19 \mathrm{~g}, 1.6$ $\mathrm{mmol}$ ) was added. The mixture was stirred at r.t. for $1 \mathrm{~h}$ and allowed to stand overnight. After removing the volatile components in vacuo, the adduct $\mathbf{1 1}$ was obtained.

Yield: $0.74 \mathrm{~g}$ (ca. 100\%); viscous amber oil; $R_{f}=0.53$ (EtOAc-hexane, $1: 8)$.

IR (film): 3253, 2980, 2931, 1738 s (CO), 1554, 1451, 1373, 1278, 1154, 1057, 843, 805, 750, 699, $609 \mathrm{~cm}^{-1}$.

${ }^{1} \mathrm{H}$ NMR $\left(400 \mathrm{MHz}, \mathrm{CDCl}_{3}\right): \delta=8.03(\mathrm{~d}, J=8.9 \mathrm{~Hz}, 1 \mathrm{H}), 7.75(\mathrm{~d}, J=$ $1.9 \mathrm{~Hz}, 1 \mathrm{H}), 7.58(\mathrm{~s}, 1 \mathrm{H}), 7.44(\mathrm{dd}, J=8.9,1.9 \mathrm{~Hz}, 1 \mathrm{H}), 7.30-7.40(\mathrm{~m}$, $5 \mathrm{H}, \mathrm{Ph}), 5.84$ (br s, $1 \mathrm{H}, \mathrm{NH}), 4.93-5.05\left(\mathrm{~m}, 2 \mathrm{H}, \mathrm{CHCH}_{\mathrm{a}} \mathrm{H}_{\mathrm{b}}+\mathrm{CHCH}_{\mathrm{a}} \mathrm{H}_{\mathrm{b}}\right.$ ), 4.67-4.74 (m, $\left.2 \mathrm{H}, \mathrm{CH}_{2} \mathrm{Ph}\right), 4.65$ (dd, $J=11.2,4.2 \mathrm{~Hz}, 1 \mathrm{H}, \mathrm{CHCH}_{\mathrm{a}} \mathrm{H}_{\mathrm{b}}$ ), $1.67\left(\mathrm{~s}, 9 \mathrm{H}, \mathrm{OC}\left(\mathrm{CH}_{3}\right)_{3}\right)$.

${ }^{13} \mathrm{C}$ NMR $\left(125 \mathrm{MHz}, \mathrm{CDCl}_{3}\right): \delta=148.88,136.92,134.18,130.03$, $128.75,128.48,128.20,127.94,125.45,122.16,116.90,116.36$, 114.49, 84.78, 77.08, 76.40, 55.70, 28.09.

MS (ESI): $m / z=492 / 490[\mathrm{M}+\mathrm{H}]^{+}$.

Anal. Calcd for $\mathrm{C}_{22} \mathrm{H}_{24} \mathrm{BrN}_{3} \mathrm{O}_{5}$ : C, 53.89; H, 4.93; N, 8.57. Found: C, 54.01; H, 5.00; N, 8.58.

\section{Addition of 0 -Pivaloylhydroxylamines 8a,b to Nitrovinylindoles 9a-f; General Procedure}

The hydrochloride salt of the corresponding $O$-pivaloylhydroxylamine (6 mmol) was dissolved in $\mathrm{CH}_{2} \mathrm{Cl}_{2}(20 \mathrm{~mL})$ and carefully shaken with saturated aqueous $\mathrm{NaHCO}_{3}$ solution, then the organic phase was washed with concd $\mathrm{NaCl}$ solution and dried over anhydrous $\mathrm{Na}_{2} \mathrm{SO}_{4}$. The solvent was removed in vacuo at $30{ }^{\circ} \mathrm{C}$ and the resulting $\mathrm{O}$-pivaloylhydroxylamine (ca. $5.2 \mathrm{mmol}$ ) was carefully mixed with nitrovinylindole $(3.5 \mathrm{mmol})$ in a round-bottom vial and allowed to stand overnight at r.t. The reaction mixture was triturated with hexane ( 7 $\mathrm{mL}$ ) and the resulting crystals of adducts $\mathbf{7 a - h}$ were filtered, washed with cold hexane $(2 \times 4 \mathrm{~mL})$, and dried in vacuo.

tert-Butyl 3-\{1-[(Pivaloyloxy)amino]-2-nitroethyl\}-1H-indole-1carboxylate (7a)

Yield: $1.33 \mathrm{~g}$ (94\%); light-brown solid; $\mathrm{mp} 109-111{ }^{\circ} \mathrm{C}$ (hexane); $R_{f}=$ 0.46 (EtOAc-hexane, 1:8).

IR (film): 3229, 2977, 2936, 1746 s (CO), 1727 s (CO), 1555, 1452, $1371,1278,1235,1154,1085,822,762,661,594 \mathrm{~cm}^{-1}$. 
${ }^{1} \mathrm{H}$ NMR $\left(400 \mathrm{MHz}, \mathrm{CDCl}_{3}\right): \delta=8.19(\mathrm{~d}, J=8.1 \mathrm{~Hz}, 1 \mathrm{H}), 7.89(\mathrm{~d}, J=$ $3.5 \mathrm{~Hz}, 1 \mathrm{H}, \mathrm{NH}), 7.70$ (d, $J=7.7 \mathrm{~Hz}, 1 \mathrm{H}), 7.67$ (s, $1 \mathrm{H}$ ), 7.39 (dd, $J=8.1$, $7.3 \mathrm{~Hz}, 1 \mathrm{H}), 7.31(\mathrm{dd}, J=7.7,7.3 \mathrm{~Hz}, 1 \mathrm{H}), 5.14-5.26(\mathrm{~m}, 1 \mathrm{H}, \mathrm{CH}-$ $\mathrm{CH}_{\mathrm{a}} \mathrm{H}_{\mathrm{b}}$ ), 5.01 (dd, $\left.J=12.9,8.0 \mathrm{~Hz}, 1 \mathrm{H}, \mathrm{CHCH}_{\mathrm{a}} \mathrm{H}_{\mathrm{b}}\right), 4.75$ (dd, $J=12.9$, $\left.4.5 \mathrm{~Hz}, 1 \mathrm{H}, \mathrm{CHCH}_{\mathrm{a}} \mathrm{H}_{\mathrm{b}}\right), 1.68\left(\mathrm{~s}, 9 \mathrm{H}, \mathrm{OC}\left(\mathrm{CH}_{3}\right)_{3}\right), 1.24\left(\mathrm{~s}, 9 \mathrm{H}, \mathrm{C}\left(\mathrm{CH}_{3}\right)_{3}\right)$.

${ }^{13} \mathrm{C}$ NMR $\left(100 \mathrm{MHz}, \mathrm{CDCl}_{3}\right): \delta=178.21,149.15,135.48,127.82$, 125.36, 124.60, 123.23, 118.99, 115.61, 113.38, 84.51, 78.04, 56.25, 38.44, 28.15 (3C), 26.90 (3C).

MS (ESI): $m / z=406[\mathrm{M}+\mathrm{H}]^{+}, 345\left[\mathrm{M}-\mathrm{CH}_{3} \mathrm{NO}_{2}+\mathrm{H}\right]^{+}, 304[\mathrm{M}-$ $\left.\left(\mathrm{CH}_{3}\right)_{3} \mathrm{CO}_{2} \mathrm{H}+\mathrm{H}\right]^{+}$.

Anal. Calcd for $\mathrm{C}_{20} \mathrm{H}_{27} \mathrm{~N}_{3} \mathrm{O}_{6}$ : C, 59.25; $\mathrm{H}, 6.71 ; \mathrm{N}, 10.36$. Found: $\mathrm{C}$, 59.31; H, 6.73; N, 10.11 .

\section{tert-Butyl 3-\{1-[(Pivaloyloxy)amino]-2-nitroethyl\}-5-bromo-1H- indole-1-carboxylate (7b)}

Yield: $1.62 \mathrm{~g}$ (96\%); white solid; $\mathrm{mp} 108-109{ }^{\circ} \mathrm{C}$ (hexane); $R_{f}=0.39$ (EtOAc-hexane, 1:8).

IR (film): 3260, 2974, 2935, 1728 s (CO), 1557, 1453, 1378, 1276, $1157,1092,1057,872,805,778,662,604 \mathrm{~cm}^{-1}$.

${ }^{1} \mathrm{H}$ NMR $\left(400 \mathrm{MHz}, \mathrm{CDCl}_{3}\right): \delta=8.07(\mathrm{~d}, J=8.8 \mathrm{~Hz}, 1 \mathrm{H}), 7.84(\mathrm{~d}, J=$ $1.8 \mathrm{~Hz}, 1 \mathrm{H}$ ), $7.66(\mathrm{~s}, 1 \mathrm{H}), 7.47$ (dd, $J=8.8,1.8 \mathrm{~Hz}, 1 \mathrm{H}), 7.89$ (br s, $1 \mathrm{H}$, $\mathrm{NH}$ ), 5.11 (dd, $\left.J=7.7,4.8 \mathrm{~Hz}, 1 \mathrm{H}, \mathrm{CHCH}_{\mathrm{a}} \mathrm{H}_{\mathrm{b}}\right), 4.99$ (dd, $J=12.8,7.7 \mathrm{~Hz}$, $\left.1 \mathrm{H}, \mathrm{CHCH}_{\mathrm{a}} \mathrm{H}_{\mathrm{b}}\right), 4.72\left(\mathrm{dd}, J=12.8,4.8 \mathrm{~Hz}, 1 \mathrm{H}, \mathrm{CHCH}_{\mathrm{a}} \mathrm{H}_{\mathrm{b}}\right), 1.67(\mathrm{~s}, 9 \mathrm{H}$, $\left.\mathrm{OC}\left(\mathrm{CH}_{3}\right)_{3}\right), 1.24\left(\mathrm{~s}, 9 \mathrm{H}, \mathrm{C}(\mathrm{O}) \mathrm{C}\left(\mathrm{CH}_{3}\right)_{3}\right)$.

${ }^{13} \mathrm{C}$ NMR $\left(100 \mathrm{MHz}, \mathrm{CDCl}_{3}\right): \delta=178.26,148.84,134.37,129.59$, 128.39, 125.86, 121.98, 117.14, 116.75, 112.91, 85.13, 77.87, 56.20, 38.54, 28.19 (3C), 27.00 (3C).

MS (ESI): $m / z=486 / 484[\mathrm{M}+\mathrm{H}]^{+}, 425 / 423\left[\mathrm{M}-\mathrm{CH}_{3} \mathrm{NO}_{2}+\mathrm{H}\right]^{+}$, $384 / 382\left[\mathrm{M}-\left(\mathrm{CH}_{3}\right)_{3} \mathrm{CO}_{2} \mathrm{H}+\mathrm{H}\right]^{+}$.

Anal. Calcd for $\mathrm{C}_{20} \mathrm{H}_{26} \mathrm{BrN}_{3} \mathrm{O}_{6}$ : C, 49.60; H, 5.41; N, 8.68. Found: C, 49.71; H, 5.50; N, 8.53.

\section{tert-Butyl 3-\{1-[(Pivaloyloxy)(methyl)amino]-2-nitroethyl\}-1H- indole-1-carboxylate (7c)}

Yield: $1.42 \mathrm{~g}$ (97\%); light-beige solid; $\mathrm{mp} 113^{\circ} \mathrm{C}$ (hexane); $R_{f}=0.47$ (EtOAc-hexane, 1:8).

IR (film): 2977, 2931, 1754 s (CO), 1737 s (CO), 1557, 1452, 1378, $1248,1153,1103,1075,1026,845,750,702,659 \mathrm{~cm}^{-1}$.

${ }^{1} \mathrm{H}$ NMR $\left(400 \mathrm{MHz}, \mathrm{CDCl}_{3}\right): \delta=8.18(\mathrm{~d}, J=7.7 \mathrm{~Hz}, 1 \mathrm{H}), 7.81(\mathrm{~d}, J=$ $7.7 \mathrm{~Hz}, 1 \mathrm{H}), 7.69(\mathrm{~s}, 1 \mathrm{H}), 7.38$ (dd, $J=8.1,7.3 \mathrm{~Hz}, 1 \mathrm{H}), 7.31$ (dd, $J=$ 7.8, 7.7 Hz, $1 \mathrm{H}$ ), 5.08 (dd, $J=7.4,5.6 \mathrm{~Hz}, 1 \mathrm{H}, \mathrm{CHCH}_{\mathrm{a}} \mathrm{H}_{\mathrm{b}}$ ), $5.00(\mathrm{dd}, J=$ 12.7, 7.4 Hz, $\left.1 \mathrm{H}, \mathrm{CHCH}_{\mathrm{a}} \mathrm{H}_{\mathrm{b}}\right), 4.65$ (dd, $J=12.7,5.6 \mathrm{~Hz}, 1 \mathrm{H}, \mathrm{CHCH}_{\mathrm{a}} \mathrm{H}_{\mathrm{b}}$ ), $2.68\left(\mathrm{~s}, 3 \mathrm{H}, \mathrm{NCH}_{3}\right), 1.69\left(\mathrm{~s}, 9 \mathrm{H}, \mathrm{OC}\left(\mathrm{CH}_{3}\right)_{3}\right), 1.26\left(\mathrm{~s}, 9 \mathrm{H}, \mathrm{C}(\mathrm{O}) \mathrm{C}\left(\mathrm{CH}_{3}\right)_{3}\right)$.

${ }^{13} \mathrm{C}$ NMR $\left(100 \mathrm{MHz}, \mathrm{CDCl}_{3}\right): \delta=175.96,149.16,135.30,128.65$, 125.22, 124.99, 123.24, 119.51, 115.36, 113.78, 84.38, 77.17, 62.04, 43.84, 38.67, 28.10 (3C), 27.05 (3C).

MS (ESI): $m / z=420[\mathrm{M}+\mathrm{H}]^{+}, 318\left[\mathrm{M}-\left(\mathrm{CH}_{3}\right)_{3} \mathrm{CO}_{2} \mathrm{H}+\mathrm{H}\right]^{+}$.

Anal. Calcd for $\mathrm{C}_{21} \mathrm{H}_{29} \mathrm{~N}_{3} \mathrm{O}_{6}: \mathrm{C}, 60.13 ; \mathrm{H}, 6.97 ; \mathrm{N}, 10.02$. Found: C, 60.27; H, 7.03; N, 10.00 .

\section{tert-Butyl 3-\{1-[(Pivaloyloxy)(methyl)amino]-2-nitroethyl\}-5-} bromo-1H-indole-1-carboxylate (7d)

Yield: $1.55 \mathrm{~g}$ (89\%); amorphous amber solid; $R_{f}=0.53$ (EtOAc-hexane, $1: 8)$.

IR (film): 2977, 2933, 1744 s (CO), 1602, 1559, 1451, 1370, 1276, $1153,1092,1057,842,807,767,679,609 \mathrm{~cm}^{-1}$.
${ }^{1} \mathrm{H}$ NMR $\left(600 \mathrm{MHz}, \mathrm{CDCl}_{3}\right): \delta=8.07(\mathrm{br} \mathrm{s}, 1 \mathrm{H}), 7.93(\mathrm{~d}, J=1.7 \mathrm{~Hz}, 1 \mathrm{H})$, $7.68(\mathrm{~s}, 1 \mathrm{H}), 7.47(\mathrm{dd}, J=9.0,1.7 \mathrm{~Hz}, 1 \mathrm{H}), 4.95-5.03\left(\mathrm{~m}, 2 \mathrm{H}, \mathrm{CHCH}_{\mathrm{a}} \mathrm{H}_{\mathrm{b}}\right.$ $\left.+\mathrm{CHCH}_{\mathrm{a}} \mathrm{H}_{\mathrm{b}}\right), 4.63\left(\mathrm{dd}, J=11.6,5.0 \mathrm{~Hz}, 1 \mathrm{H}, \mathrm{CHCH}_{\mathrm{a}} \mathrm{H}_{\mathrm{b}}\right), 2.67(\mathrm{~s}, 3 \mathrm{H}$, $\left.\mathrm{NCH}_{3}\right), 1.68\left(\mathrm{~s}, 9 \mathrm{H}, \mathrm{OC}\left(\mathrm{CH}_{3}\right)_{3}\right), 1.26\left(\mathrm{~s}, 9 \mathrm{H}, \mathrm{C}(\mathrm{O}) \mathrm{C}_{(}\left(\mathrm{CH}_{3}\right)_{3}\right)$.

${ }^{13} \mathrm{C}$ NMR $\left(100 \mathrm{MHz}, \mathrm{CDCl}_{3}\right): \delta=175.79,148.76,134.11,130.37$, 128.20, 126.17, 122.20, 116.87, 116.71, 113.16, 84.94, 77.00, 61.86, 43.83, 38.66, 28.08 (3C), 27.07 (3C).

MS (ESI): $m / z=500 / 498[\mathrm{M}+\mathrm{H}]^{+}, 398 / 396\left[\mathrm{M}-\left(\mathrm{CH}_{3}\right)_{3} \mathrm{CO}_{2} \mathrm{H}+\mathrm{H}\right]^{+}$.

Anal. Calcd for $\mathrm{C}_{21} \mathrm{H}_{28} \mathrm{BrN}_{3} \mathrm{O}_{6}$ : C, 50.61; H, 5.66; N, 8.43. Found: C, 50.90; H, 5.81; N, 8.25.

\section{tert-Butyl 3-\{1-[(Pivaloyloxy)(methyl)amino]-2-nitroethyl\}-6-} bromo-1H-indole-1-carboxylate (7e)

Yield: $1.70 \mathrm{~g}$ (98\%); light-beige solid; $\mathrm{mp} 115-116{ }^{\circ} \mathrm{C}$ (hexane); $R_{f}=$ 0.39 (EtOAc-hexane, 1:8).

IR (film): 2976, 2933, 1762 s (CO), 1727 s (CO), 1561, 1433, 1370, $1255,1152,1097,865,818,772,679,590 \mathrm{~cm}^{-1}$.

${ }^{1} \mathrm{H}$ NMR $\left(600 \mathrm{MHz}, \mathrm{CDCl}_{3}\right): \delta=8.40(\mathrm{~s}, 1 \mathrm{H}), 7.70(\mathrm{~d}, J=8.3 \mathrm{~Hz}, 1 \mathrm{H})$, $7.64(\mathrm{~s}, 1 \mathrm{H}), 7.42(\mathrm{dd}, J=8.3,1.7 \mathrm{~Hz}, 1 \mathrm{H}), 5.02(\mathrm{dd}, J=7.4,5.8 \mathrm{~Hz}, 1 \mathrm{H}$, $\left.\mathrm{CHCH}_{\mathrm{a}} \mathrm{H}_{\mathrm{b}}\right), 4.98\left(\mathrm{dd}, J=12.4,7.4 \mathrm{~Hz}, 1 \mathrm{H}, \mathrm{CHCH}_{\mathrm{a}} \mathrm{H}_{\mathrm{b}}\right), 4.63(\mathrm{dd}, J=12.7$, $\left.5.8 \mathrm{~Hz}, 1 \mathrm{H}, \mathrm{CHCH}_{\mathrm{a}} \mathrm{H}_{\mathrm{b}}\right), 2.66\left(\mathrm{~s}, 3 \mathrm{H}, \mathrm{NCH}_{3}\right), 1.69\left(\mathrm{~s}, 9 \mathrm{H}, \mathrm{OC}\left(\mathrm{CH}_{3}\right)_{3}\right), 1.26$ $\left(\mathrm{s}, 9 \mathrm{H}, \mathrm{C}(\mathrm{O}) \mathrm{C}\left(\mathrm{CH}_{3}\right)_{3}\right)$.

${ }^{13} \mathrm{C}$ NMR $\left(100 \mathrm{MHz}, \mathrm{CDCl}_{3}\right): \delta=175.92,148.72,136.02,127.39$, 126.53, 125.29, 120.84, 119.09, 118.61, 113.91, 85.01, 77.00, 62.02, 43.70, 38.64, $28.03(3 \mathrm{C}), 27.02$ (3C).

MS (ESI): $m / z=522 / 520[\mathrm{M}+\mathrm{Na}]^{+}$.

Anal. Calcd for $\mathrm{C}_{21} \mathrm{H}_{28} \mathrm{BrN}_{3} \mathrm{O}_{6}$ : C, 50.61; H, 5.66; N, 8.43. Found: C, 50.73; H, 5.72; N, 8.42.

tert-Butyl 3-\{1-[(Pivaloyloxy)(methyl)amino]-2-nitroethyl\}-5methoxy-1H-indole-1-carboxylate (7f)

Yield: 1.43 (91\%); yellow solid; $\mathrm{mp} 96{ }^{\circ} \mathrm{C}$ (hexane); $R_{f}=0.33$ (EtOAchexane, $1: 8)$.

IR (film): 2971, 2935, 1755 s (CO), 1731 s (CO), 1557, 1480, 1382, $1286,1157,1098,1070,849,807,767,677,626 \mathrm{~cm}^{-1}$.

${ }^{1} \mathrm{H} \mathrm{NMR}\left(600 \mathrm{MHz}, \mathrm{CDCl}_{3}\right): \delta=8.04(\mathrm{br} \mathrm{s}, 1 \mathrm{H}), 7.64(\mathrm{~s}, 1 \mathrm{H}), 7.28(\mathrm{~d}, J=$ $1.7 \mathrm{~Hz}, 1 \mathrm{H}), 6.97(\mathrm{dd}, J=9.0,1.7 \mathrm{~Hz}, 1 \mathrm{H}), 5.02(\mathrm{dd}, J=7.4,5.0 \mathrm{~Hz}, 1 \mathrm{H}$, $\left.\mathrm{CHCH}_{\mathrm{a}} \mathrm{H}_{\mathrm{b}}\right), 4.98\left(\mathrm{dd}, J=12.4,7.4 \mathrm{~Hz}, 1 \mathrm{H}, \mathrm{CHCH}_{\mathrm{a}} \mathrm{H}_{\mathrm{b}}\right), 4.63(\mathrm{dd}, J=12.4$, $\left.5.0 \mathrm{~Hz}, 1 \mathrm{H}, \mathrm{CHCH}_{\mathrm{a}} \mathrm{H}_{\mathrm{b}}\right), 3.90\left(\mathrm{~s}, 3 \mathrm{H}, \mathrm{OCH}_{3}\right), 2.68\left(\mathrm{~s}, 3 \mathrm{H}, \mathrm{NCH}_{3}\right), 1.67(\mathrm{~s}$, $\left.9 \mathrm{H}, \mathrm{OC}\left(\mathrm{CH}_{3}\right)_{3}\right), 1.25\left(\mathrm{~s}, 9 \mathrm{H}, \mathrm{C}(\mathrm{O}) \mathrm{C}\left(\mathrm{CH}_{3}\right)_{3}\right)$.

${ }^{13} \mathrm{C}$ NMR $\left(100 \mathrm{MHz}, \mathrm{CDCl}_{3}\right): \delta=175.89,156.27,149.14,130.00$, 129.53, 125.39, 116.13, 114.07, 113.70, 102.11, 84.21, 77.00, 62.13, $55.78,43.71,38.66,28.12(3 \mathrm{C}), 27.06(3 \mathrm{C})$.

MS (ESI): $m / z=450[\mathrm{M}+\mathrm{H}]^{+}, 348\left[\mathrm{M}-\left(\mathrm{CH}_{3}\right)_{3} \mathrm{CO}_{2} \mathrm{H}+\mathrm{H}\right]^{+}$.

Anal. Calcd for $\mathrm{C}_{22} \mathrm{H}_{31} \mathrm{~N}_{3} \mathrm{O}_{7}$ : C, 58.78; $\mathrm{H}, 6.95 ; \mathrm{N}, 9.35$. Found: C, 58.89; H, 7.00; N, 9.23.

\section{tert-Butyl 3-\{1-[(Pivaloyloxy)(methyl)amino]-2-nitroethyl\}-6-} chloro-1H-indole-1-carboxylate $(7 \mathrm{~g})$

Yield: 1.54 (97\%); light-brown solid; $\mathrm{mp} 108-109{ }^{\circ} \mathrm{C}$ (hexane); $R_{f}=$ 0.39 (EtOAc-hexane, 1:8).

IR (film): 2976, 2906, $1754 \mathrm{~s}$ (CO), $1744 \mathrm{~s}$ (CO), 1562, 1439, 1368, $1256,1157,1100,866,817,761,661,600 \mathrm{~cm}^{-1}$.

${ }^{1} \mathrm{H}$ NMR $\left(400 \mathrm{MHz}, \mathrm{CDCl}_{3}\right): \delta=8.21(\mathrm{~s}, 1 \mathrm{H}), 7.75(\mathrm{~d}, J=8.5 \mathrm{~Hz}, 1 \mathrm{H})$, $7.65(\mathrm{~s}, 1 \mathrm{H}), 7.27$ (dd, J = 8.5, 1.7 Hz, $1 \mathrm{H}), 4.92-5.02\left(\mathrm{~m}, 2 \mathrm{H}, \mathrm{CHCH}_{\mathrm{a}} \mathrm{H}_{\mathrm{b}}\right.$ $\left.+\mathrm{CHCH}_{\mathrm{a}} \mathrm{H}_{\mathrm{b}}\right), 4.63\left(\mathrm{dd}, J=12.1,5.3 \mathrm{~Hz}, 1 \mathrm{H}, \mathrm{CHCH}_{\mathrm{a}} \mathrm{H}_{\mathrm{b}}\right), 2.66(\mathrm{~s}, 3 \mathrm{H}$,

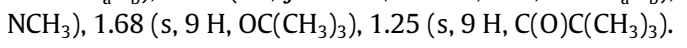


${ }^{13} \mathrm{C}$ NMR (100 MHz, $\left.\mathrm{CDCl}_{3}\right): \delta=175.91,148.75,135.75,131.35$, 127.04, 125.38, 123.86, 120.53, 115.70, 113.92, 84.98, 77.00, 62.09, 43.68, 38.65, $28.04(3 \mathrm{C}), 27.02$ (3C).

MS (ESI): $m / z=456 / 454[\mathrm{M}+\mathrm{H}]^{+}, 354 / 352\left[\mathrm{M}-\left(\mathrm{CH}_{3}\right)_{3} \mathrm{CO}_{2} \mathrm{H}+\mathrm{H}\right]^{+}$.

Anal. Calcd for $\mathrm{C}_{21} \mathrm{H}_{28} \mathrm{ClN}_{3} \mathrm{O}_{6}$ : C, 55.57; H, 6.22; N, 9.26. Found: C, $55.69 ; \mathrm{H}, 6.28 ; \mathrm{N}, 9.21$.

\section{1-Acetyl-3-\{1-[(pivaloyloxy)(methyl)amino]-2-nitroethyl\}-1H-in- dole (7h)}

Yield: $1.11 \mathrm{~g}$ (88\%); light-orange solid; $\mathrm{mp} 84-85^{\circ} \mathrm{C}$ (hexane); $R_{f}=$ 0.15 (EtOAc-hexane, 1:8).

IR (film): 2973, 2937, 2910, 2874, 1753 s (CO), 1715 s (CO), 1553, $1451,1386,1313,1225,1125,1077,1023,939,755,672,641$, $564 \mathrm{~cm}^{-1}$.

${ }^{1} \mathrm{H}$ NMR $\left(600 \mathrm{MHz}, \mathrm{CDCl}_{3}\right): \delta=8.44(\mathrm{br} \mathrm{d}, J=8.3 \mathrm{~Hz}, 1 \mathrm{H}), 7.83(\mathrm{~d}, J=$ $7.4 \mathrm{~Hz}, 1 \mathrm{H}), 7.55$ (s, $1 \mathrm{H}), 7.38-7.43(\mathrm{~m}, 1 \mathrm{H}), 7.32-7.37(\mathrm{~m}, 1 \mathrm{H}), 5.07$ $\left(\mathrm{dd}, J=7.4,5.8 \mathrm{~Hz}, 1 \mathrm{H}, \mathrm{CHCH}_{\mathrm{a}} \mathrm{H}_{\mathrm{b}}\right), 4.98(\mathrm{dd}, J=12.8,7.4 \mathrm{~Hz}, 1 \mathrm{H}, \mathrm{CH}-$ $\left.\mathrm{CH}_{\mathrm{a}} \mathrm{H}_{\mathrm{b}}\right), 4.66\left(\mathrm{dd}, \mathrm{J}=12.8,5.8 \mathrm{~Hz}, 1 \mathrm{H}, \mathrm{CHCH}_{\mathrm{a}} \mathrm{H}_{\mathrm{b}}\right), 2.70\left(\mathrm{~s}, 3 \mathrm{H}, \mathrm{C}(\mathrm{O}) \mathrm{CH}_{3}\right.$ or $\left.\mathrm{NCH}_{3}\right), 2.66\left(\mathrm{~s}, 3 \mathrm{H}, \mathrm{C}(\mathrm{O}) \mathrm{CH}_{3}\right.$ or $\left.\mathrm{NCH}_{3}\right), 1.25\left(\mathrm{~s}, 9 \mathrm{H}, \mathrm{C}(\mathrm{O}) \mathrm{C}\left(\mathrm{CH}_{3}\right)_{3}\right)$.

${ }^{13} \mathrm{C}$ NMR $\left(100 \mathrm{MHz}, \mathrm{CDCl}_{3}\right): \delta=175.98,168.27,135.76,128.36$, $126.16,124.28,124.22,119.58,116.69,116.11,77.14,62.39,44.09$, $38.68,27.00$ (3C), 23.92.

MS (ESI): $m / z=362[\mathrm{M}+\mathrm{H}]^{+}, 260\left[\mathrm{M}-\left(\mathrm{CH}_{3}\right)_{3} \mathrm{CO}_{2} \mathrm{H}+\mathrm{H}\right]^{+}$.

Anal. Calcd for $\mathrm{C}_{18} \mathrm{H}_{23} \mathrm{~N}_{3} \mathrm{O}_{5}$ : C, 59.82; $\mathrm{H}, 6.41 ; \mathrm{N}, 11.63$. Found: $\mathrm{C}$, 60.00; H, 6.48; N, 11.58 .

\section{Catalytic Hydrogenation of Adduct 11}

To a solution of adduct $11(0.54 \mathrm{~g}, 1.1 \mathrm{mmol})$ in $\mathrm{MeOH}(10 \mathrm{~mL})$ was added $\mathrm{AcOH}$ (9.5 mL, $1.165 \mathrm{~mol})$ and 5\% Pd on charcoal $(0.05 \mathrm{~g})$ and the mixture was purged with hydrogen. The mixture was vigorously stirred under a hydrogen atmosphere ( $1 \mathrm{~atm})$ for $18 \mathrm{~h}$, filtered, and concentrated in vacuo. The residue was dissolved in $\mathrm{CH}_{2} \mathrm{Cl}_{2}(50 \mathrm{~mL})$, treated with cold $\mathrm{NaOH}$ solution $(10 \%, 40 \mathrm{~mL})$ and concd $\mathrm{NaCl}$ solutions $\left(20 \mathrm{~mL}\right.$ ), and dried over anhydrous $\mathrm{Na}_{2} \mathrm{SO}_{4}$. The solvent was removed in vacuo and the residue was purified by chromatography on a column of silica gel $\left(\mathrm{CHCl}_{3}-\mathrm{MeOH}-\mathrm{NH}_{3(\mathrm{aq})}, 100: 10: 0.2\right)$ to afford diamine $\mathbf{7 b}(0.206 \mathrm{~g}, 68 \%)$. Its characteristics correspond to the sample obtained from adduct $9 \mathbf{a}$ (see below).

\section{Reduction of Adduct 11 with Zn Dust}

To a solution of adduct $\mathbf{1 1}(0.54 \mathrm{~g}, 1.1 \mathrm{mmol})$ in $\mathrm{MeOH}(10 \mathrm{~mL})$ was added $\mathrm{AcOH}(9.5 \mathrm{~mL}, 1.165 \mathrm{~mol})$ and $\mathrm{Zn}$ dust $(1.44 \mathrm{~g}, 22 \mathrm{mmol})$. The mixture was vigorously stirred at r.t. for $2 \mathrm{~h}$, and then another portion of $\mathrm{Zn}$ dust ( $1.43 \mathrm{~g}, 22 \mathrm{mmol}$ ) was added. The resulting mixture was vigorously stirred further for $12 \mathrm{~h}$, filtered, and concentrated in vacuo. The residue was dissolved in $\mathrm{CH}_{2} \mathrm{Cl}_{2}(100 \mathrm{~mL})$, treated with chipped ice (10 g), and carefully shaken with cold $\mathrm{NaOH}$ solution $(10 \%, 90 \mathrm{~mL})$. The organic layer was separated and washed with cold $\mathrm{NaOH}(10 \%, 30 \mathrm{~mL})$ and concd $\mathrm{NaCl}$ solutions $(20 \mathrm{~mL})$, and dried over anhydrous $\mathrm{Na}_{2} \mathrm{SO}_{4}$. The solvent was removed in vacuo and the residue was purified by chromatography on a column of silica gel with gradient of $\mathrm{MeOH}$ in $\mathrm{CHCl}_{3}$ to afford compound 12.

Yield: $0.27 \mathrm{~g}$ (53\%); pale-yellow oil.

IR (film): 3378 br, 2978, 2931, 2866, 1736 s, (CO), 1603, 1451, 1371, $1256,1155,1054,842,803,750,698,611 \mathrm{~cm}^{-1}$.
${ }^{1} \mathrm{H}$ NMR $\left(600 \mathrm{MHz}, \mathrm{CDCl}_{3}\right): \delta=8.03(\mathrm{~m}, 1 \mathrm{H}), 7.81(\mathrm{~d}, J=1.8 \mathrm{~Hz}, 1 \mathrm{H})$, $7.55(\mathrm{~s}, 1 \mathrm{H}), 7.41$ (dd, $J=8.8,1.8 \mathrm{~Hz}, 1 \mathrm{H}), 7.28-7.36(\mathrm{~m}, 5 \mathrm{H}, \mathrm{Ph}), 6.02$ (br s, $1 \mathrm{H}, \mathrm{NH}), 4.64-4.70\left(\mathrm{~m}, 2 \mathrm{H}, \mathrm{CH}_{2} \mathrm{Ph}\right), 4.19-4.24\left(\mathrm{~m}, 1 \mathrm{H}, \mathrm{CHCH}_{2}\right)$, 3.07-3.13 (m, $\left.2 \mathrm{H}, \mathrm{CHCH}_{2}\right), 1.67\left(\mathrm{~s}, 9 \mathrm{H}, \mathrm{OC}\left(\mathrm{CH}_{3}\right)_{3}\right), 1.47$ (br s, $2 \mathrm{H}$, $\mathrm{NH}_{2}$ ).

${ }^{13} \mathrm{C}$ NMR $\left(150 \mathrm{MHz}, \mathrm{CDCl}_{3}\right): \delta=149.20,137.62,134.28,131.10$, $128.54,128.31,127.85,127.33,124.77,122.60,118.24,116.70$, $115.90,84.18,76.73,59.96,43.26,28.12(3 \mathrm{C})$.

MS (ESI): $m / z=462 / 460[\mathrm{M}+\mathrm{H}]^{+}$.

Anal. Calcd for $\mathrm{C}_{22} \mathrm{H}_{26} \mathrm{BrN}_{3} \mathrm{O}_{3}$ : C, 57.40; H, 5.69; N, 9.13. Found: C, 57.37; H, 5.61; N, 9.04.

After the chromatography, diamine $\mathbf{7 b}(5.8 \mathrm{mg}, 15 \%)$ was also isolated. The analytical data was in accordance with the characteristics of the sample obtained from adduct $\mathbf{9 b}$ (see below).

\section{Synthesis of (Indol-3-yl)ethane-1,2-diamines 6; General Procedure A}

A solution of adduct $7(2.2 \mathrm{mmol})$ in EtOAc $(8.3 \mathrm{~mL})$ was added to a cooled $\left(-10{ }^{\circ} \mathrm{C}\right)$ mixture of $\mathrm{MeOH}(16.5 \mathrm{~mL})$ and $\mathrm{HBr}(40 \%, 5.5 \mathrm{~mL}$, $37.8 \mathrm{mmol})$ under vigorous stirring and treated with Zn dust $(2.2 \mathrm{~g}$, $33 \mathrm{mmol})$. The reaction mixture was allowed to warm to $0-5{ }^{\circ} \mathrm{C}(1 \mathrm{~h})$, stirred at that temperature for $1 \mathrm{~h}$, and filtered. The precipitate was filtered off and rinsed with a small amount of $\mathrm{MeOH}$. The filtrate was diluted with $\mathrm{CH}_{2} \mathrm{Cl}_{2}(100 \mathrm{~mL})$, treated with crushed ice $(10 \mathrm{~g})$, and carefully shaken with cold $\mathrm{NaOH}$ solution $(10 \%, 90 \mathrm{~mL})$. The organic layer was separated and the aqueous layer was washed with $\mathrm{CH}_{2} \mathrm{Cl}_{2}$ $(20 \mathrm{~mL})$. The combined organic extracts were washed with cold $\mathrm{NaOH}$ $(10 \%, 30 \mathrm{~mL})$ and concd $\mathrm{NaCl}$ solutions $(20 \mathrm{~mL})$, and dried over anhydrous $\mathrm{Na}_{2} \mathrm{SO}_{4}$. The solvent was removed in vacuo to afford target diamine $\mathbf{6}$ as a yellowish oil. The product was pure enough for further syntheses. Chromatographic purification on a column with silica gel $\left(\mathrm{CHCl}_{3}-\mathrm{MeOH}-\mathrm{NH}_{3(\mathrm{aq})}, 100: 10: 0.2\right)$ was performed, if necessary.

\section{Synthesis of (Indol-3-yl)ethane-1,2-diamines 6; General Procedure B}

A solution of adduct $7(2.2 \mathrm{mmol})$ in EtOAc $(13 \mathrm{~mL})$ was added to a cooled $\left(-10^{\circ} \mathrm{C}\right)$ mixture of $\mathrm{MeOH}(26 \mathrm{~mL})$ and $\mathrm{HBr}(40 \%, 13 \mathrm{~mL}, 89.0$ $\mathrm{mmol}$ ) under vigorous stirring and treated with Zn dust (1.44 g, 22 $\mathrm{mmol})$. The mixture was allowed to warm to $0{ }^{\circ} \mathrm{C}(1 \mathrm{~h})$, then another portion of Zn dust ( $1.44 \mathrm{~g}, 22 \mathrm{mmol}$ ) was added. The reaction mixture was stirred further at $0-5{ }^{\circ} \mathrm{C}$ for $5 \mathrm{~h}$, and filtered. The precipitate was rinsed with a small amount of $\mathrm{MeOH}$ and the filtrate was diluted with $\mathrm{CH}_{2} \mathrm{Cl}_{2}(100 \mathrm{~mL})$, treated with crushed ice $(10 \mathrm{~g})$, and shaken carefully with cold $\mathrm{NaOH}$ solution $(10 \%, 120 \mathrm{~mL})$. General Procedure A was then followed.

1-[(1-tert-Butoxycarbonyl)-1H-indol-3-yl]ethane-1,2-diamine (6a) Obtained by following General Procedure A.

Yield: $0.51 \mathrm{~g}$ (84\%); pale-yellow viscous oil; $R_{f}=0.30\left(\mathrm{CHCl}_{3}-\mathrm{MeOH}-\right.$ $\left.\mathrm{NH}_{3(\mathrm{aq})}, 10: 1: 0.02\right)$.

IR (film): 3365 br, 2978, 2932, 2868, 1729 s (CO), 1606, 1555, 1453, $1375,1256,1158,1079,856,747,591 \mathrm{~cm}^{-1}$.

${ }^{1} \mathrm{H} \mathrm{NMR}\left(400 \mathrm{MHz}, \mathrm{CDCl}_{3}\right): \delta=8.16(\mathrm{~d}, J=8.2 \mathrm{~Hz}, 1 \mathrm{H}, 7-\mathrm{H}), 7.61(\mathrm{~d}, J=$ $7.8 \mathrm{~Hz}, 1 \mathrm{H}, 4-\mathrm{H}), 7.54$ (s, $1 \mathrm{H}, 2-\mathrm{H}), 7.32$ (dd, $J=8.2,7.1 \mathrm{~Hz}, 1 \mathrm{H}, 6-\mathrm{H}$ ), $7.23(\mathrm{dd}, J=7.8,7.1 \mathrm{~Hz}, 1 \mathrm{H}, 5-\mathrm{H}), 4.23(\mathrm{dd} J=6.6,4.5 \mathrm{~Hz}, 1 \mathrm{H}, \mathrm{CH}-$ $\mathrm{CH}_{\mathrm{a}} \mathrm{H}_{\mathrm{b}}$ ), $3.10\left(\mathrm{dd}, J=12.7,4.5 \mathrm{~Hz}, 1 \mathrm{H}, \mathrm{CHCH}_{\mathrm{a}} \mathrm{H}_{\mathrm{b}}\right), 2.94(\mathrm{dd}, J=12.7$, 6.6 Hz, $\left.1 \mathrm{H}, \mathrm{CHCH}_{\mathrm{a}} \mathrm{H}_{\mathrm{b}}\right), 1.67$ (s, $\left.9 \mathrm{H}, \mathrm{OC}\left(\mathrm{CH}_{3}\right)_{3}\right), 1.58$ (br s, $4 \mathrm{H}, 2 \mathrm{NH}_{2}$ ).

${ }^{13} \mathrm{C}$ NMR $\left(100 \mathrm{MHz}, \mathrm{CDCl}_{3}\right): \delta=149.59,135.74,128.83,124.43$, 123.34, 122.39, 122.31, 119.17, 115.34, 83.57, 50.51, 47.94, 28.09. 
MS (ESI): $m / z=276[\mathrm{M}+\mathrm{H}]^{+}, 259\left[\mathrm{M}-\mathrm{NH}_{3}+\mathrm{H}\right]^{+}$.

Anal. Calcd for $\mathrm{C}_{15} \mathrm{H}_{21} \mathrm{~N}_{3} \mathrm{O}_{2}$ : C, 65.43; H, 7.69; N, 15.26. Found: C, 65.33; H, 7.73; N, 15.15 .

\section{1-[5-Bromo-(1-tert-Butoxycarbonyl)-1H-indol-3-yl]ethane-1,2-di- amine (6b)}

Obtained by following General Procedure A.

Yield: $0.75 \mathrm{~g}$ (95\%); pale-yellow viscous oil; $R_{f}=0.35\left(\mathrm{CHCl}_{3}-\mathrm{MeOH}-\right.$ $\left.\mathrm{NH}_{3(\mathrm{aq})}, 10: 1: 0.02\right)$.

IR (film): 3368 br, 2979, 2933, 2867, 1733 s (CO), 1601, 1450, 1372, $1276,1255,1157,1055,842,801,765,636 \mathrm{~cm}^{-1}$.

${ }^{1} \mathrm{H} \mathrm{NMR}\left(400 \mathrm{MHz}, \mathrm{CDCl}_{3}\right): \delta=8.02(\mathrm{~d}, J=8.8 \mathrm{~Hz}, 1 \mathrm{H}, 7-\mathrm{H}), 7.76(\mathrm{~d}, J=$ $2.0 \mathrm{~Hz}, 1 \mathrm{H}, 4-\mathrm{H}), 7.54(\mathrm{~s}, 1 \mathrm{H}, 2-\mathrm{H}), 7.39$ (dd, $J=8.8,2.0 \mathrm{~Hz}, 1 \mathrm{H}, 6-\mathrm{H})$, $4.18\left(\mathrm{dd} J=7.0,4.7 \mathrm{~Hz}, 1 \mathrm{H}, \mathrm{CHCH}_{\mathrm{a}} \mathrm{H}_{\mathrm{b}}\right), 3.09(\mathrm{dd}, J=12.7,4.7 \mathrm{~Hz}, 1 \mathrm{H}$, $\left.\mathrm{CHCH}_{\mathrm{a}} \mathrm{H}_{\mathrm{b}}\right), 2.91\left(\mathrm{dd}, J=12.7,7.0 \mathrm{~Hz}, 1 \mathrm{H}, \mathrm{CHCH}_{\mathrm{a}} \mathrm{H}_{\mathrm{b}}\right), 1.67(\mathrm{br} \mathrm{s}, 4 \mathrm{H}$, $\left.2 \mathrm{NH}_{2}\right), 1.66\left(\mathrm{~s}, 9 \mathrm{H}, \mathrm{OC}\left(\mathrm{CH}_{3}\right)_{3}\right)$.

${ }^{13} \mathrm{C}$ NMR $\left(100 \mathrm{MHz}, \mathrm{CDCl}_{3}\right): \delta=149.29,134.60,130.69,127.29$, $123.54,122.82,122.11,116.83,115.84,84.08,50.55,48.13,28.14$ (3C).

MS (ESI): $m / z=356 / 354[\mathrm{M}+\mathrm{H}]^{+}, 339 / 337\left[\mathrm{M}-\mathrm{NH}_{3}+\mathrm{H}\right]^{+}$.

Anal. Calcd for $\mathrm{C}_{15} \mathrm{H}_{20} \mathrm{BrN}_{3} \mathrm{O}_{2}$ : C, 50.86; H, 5.69; N, 11.86. Found: $\mathrm{C}$, 50.80; H, 5.75; N, 11.79 .

\section{1-[(1-tert-Butoxycarbonyl)-1H-indol-3-yl]- $N^{1}$-methylethane-1,2- diamine (6c)}

Obtained by following General Procedure B.

Yield: $0.59 \mathrm{~g}$ (93\%); pale-yellow viscous oil; $R_{f}=0.34\left(\mathrm{CHCl}_{3}-\mathrm{MeOH}-\right.$ $\left.\mathrm{NH}_{3(\mathrm{aq})}, 10: 1: 0.02\right)$.

IR (film): 3366 br, 2976, 2932, 2794, 1728 s (CO), 1607, 1450, 1367, $1250,1152,1078,1017,854,743,665 \mathrm{~cm}^{-1}$.

${ }^{1} \mathrm{H} \mathrm{NMR}\left(400 \mathrm{MHz}, \mathrm{CDCl}_{3}\right): \delta=8.17(\mathrm{~d}, J=8.0 \mathrm{~Hz}, 1 \mathrm{H}, 7-\mathrm{H}), 7.66(\mathrm{~d}, J=$ $7.7 \mathrm{~Hz}, 1 \mathrm{H}, 4-\mathrm{H}), 7.53(\mathrm{~s}, 1 \mathrm{H}, 2-\mathrm{H}), 7.33$ (dd, $J=8.0,7.4 \mathrm{~Hz}, 1 \mathrm{H}, 6-\mathrm{H})$, 7.23 (dd, $J=7.7,7.4 \mathrm{~Hz}, 1 \mathrm{H}, 5-\mathrm{H}$ ), 3.83 (dd $J=6.4,5.4 \mathrm{~Hz}, 1 \mathrm{H}, \mathrm{CH}-$ $\left.\mathrm{CH}_{\mathrm{a}} \mathrm{H}_{\mathrm{b}}\right), 3.10\left(\mathrm{dd}, J=12.7,5.4 \mathrm{~Hz}, 1 \mathrm{H}, \mathrm{CHCH}_{\mathrm{a}} H_{\mathrm{b}}\right), 2.98(\mathrm{dd}, J=12.7$, $\left.6.4 \mathrm{~Hz}, 1 \mathrm{H}, \mathrm{CHCH}_{\mathrm{a}} \mathrm{H}_{\mathrm{b}}\right), 2.43\left(\mathrm{~s}, 3 \mathrm{H}, \mathrm{NCH}_{3}\right), 1.68\left(\mathrm{~s}, 9 \mathrm{H}, \mathrm{OC}\left(\mathrm{CH}_{3}\right)_{3}\right), 1.43$ (br s, $3 \mathrm{H}, \mathrm{NH}+\mathrm{NH}_{2}$ ).

${ }^{13} \mathrm{C}$ NMR (100 MHz, $\left.\mathrm{CDCl}_{3}\right): \delta=149.48,135.68,129.27,124.25$, 123.12, 122.23, 120.92, 119.33, 115.19, 83.39, 59.72, 46.04, 34.45, $28.00(3 \mathrm{C})$.

MS (ESI): $m / z=290[\mathrm{M}+\mathrm{H}]^{+}$.

Anal. Calcd for $\mathrm{C}_{16} \mathrm{H}_{23} \mathrm{~N}_{3} \mathrm{O}_{2}$ : C, 66.41; $\mathrm{H}, 8.01 ; \mathrm{N}, 14.52$. Found: $\mathrm{C}$, 66.33; H, 8.06; N, 14.45 .

\section{1-[5-Bromo-(1-tert-butoxycarbonyl)-1H-indol-3-yl]- $N^{1}$-methyl- ethane-1,2-diamine (6d)}

Obtained by following General Procedure B.

Yield: $0.70 \mathrm{~g}$ (86\%); pale-yellow viscous oil; $R_{f}=0.46\left(\mathrm{CHCl}_{3}-\mathrm{MeOH}-\right.$ $\left.\mathrm{NH}_{3(\mathrm{aq})}, 10: 1: 0.02\right)$.

IR (film): 3326 br, 2934, 2791, 1733 s (CO), 1602, 1449, 1373, 1275, $1256,1156,1055,802,732,640,611 \mathrm{~cm}^{-1}$.

${ }^{1} \mathrm{H}$ NMR (400 MHz, CDCl $): \delta=8.01(\mathrm{~d}, J=8.7 \mathrm{~Hz}, 1 \mathrm{H}, 7-\mathrm{H}), 7.81(\mathrm{~d}, J=$ $1.9 \mathrm{~Hz}, 1 \mathrm{H}, 4-\mathrm{H}), 7.52(\mathrm{~s}, 1 \mathrm{H}, 2-\mathrm{H}), 7.37$ (dd, $J=8.7,1.9 \mathrm{~Hz}, 1 \mathrm{H}, 6-\mathrm{H})$, $3.76\left(\mathrm{dd} J=6.7,5.3 \mathrm{~Hz}, 1 \mathrm{H}, \mathrm{CHCH}_{\mathrm{a}} \mathrm{H}_{\mathrm{b}}\right), 3.06(\mathrm{dd}, J=12.8,5.3 \mathrm{~Hz}, 1 \mathrm{H}$, $\left.\mathrm{CHCH}_{\mathrm{a}} \mathrm{H}_{\mathrm{b}}\right), 2.93\left(\mathrm{dd}, J=12.8,6.7 \mathrm{~Hz}, 1 \mathrm{H}, \mathrm{CHCH}_{\mathrm{a}} \mathrm{H}_{\mathrm{b}}\right), 2.39(\mathrm{~s}, 3 \mathrm{H}$, $\left.\mathrm{NCH}_{3}\right), 1.69$ (br s, $\left.3 \mathrm{H}, \mathrm{NH}+\mathrm{NH}_{2}\right), 1.64\left(\mathrm{~s}, 9 \mathrm{H}, \mathrm{OC}\left(\mathrm{CH}_{3}\right)_{3}\right)$.
${ }^{13} \mathrm{C}$ NMR $\left(100 \mathrm{MHz}, \mathrm{CDCl}_{3}\right): \delta=149.19,134.55,131.07,127.18$, $124.42,122.24,120.28,116.71,115.77,84.01,59.62,46.05,34.42$, $28.07(3 \mathrm{C})$.

MS (ESI): $m / z=370 / 368[\mathrm{M}+\mathrm{H}]^{+}$.

Anal. Calcd for $\mathrm{C}_{16} \mathrm{H}_{22} \mathrm{BrN}_{3} \mathrm{O}_{2}$ : C, 52.18; $\mathrm{H}, 6.02 ; \mathrm{N}, 11.41$. Found: $\mathrm{C}$, 52.20; H, 6.06; N, 11.34 .

1-[6-Bromo-(1-tert-butoxycarbonyl)-1H-indol-3-yl]- $N^{1}$-methylethane-1,2-diamine $(6 e)$

Obtained by following General Procedure B.

Yield: $0.74 \mathrm{~g}$ (92\%); pale-yellow viscous oil; $R_{f}=0.31\left(\mathrm{CHCl}_{3}-\mathrm{MeOH}-\right.$ $\left.\mathrm{NH}_{3(\mathrm{aq})}, 10: 1: 0.02\right)$.

IR (film): 3320 br, 2977, 2935, 2792, 1736 s (CO), 1602, 1453, 1432, $1370,1251,1156,1082,810,766,590 \mathrm{~cm}^{-1}$.

${ }^{1} \mathrm{H} \mathrm{NMR}\left(400 \mathrm{MHz}, \mathrm{CDCl}_{3}\right): \delta=8.37$ (br s, $\left.1 \mathrm{H}, 7-\mathrm{H}\right), 7.55(\mathrm{~d}, J=8.4 \mathrm{~Hz}$, $1 \mathrm{H}, 4-\mathrm{H}), 7.50(\mathrm{~s}, 1 \mathrm{H}, 2-\mathrm{H}), 7.33$ (dd, $J=8.4,1.7 \mathrm{~Hz}, 1 \mathrm{H}, 5-\mathrm{H}), 3.81$ (dd $\left.J=6.8,5.3 \mathrm{~Hz}, 1 \mathrm{H}, \mathrm{CHCH}_{\mathrm{a}} \mathrm{H}_{\mathrm{b}}\right), 3.08\left(\mathrm{dd}, J=12.8,5.3 \mathrm{~Hz}, 1 \mathrm{H}, \mathrm{CHCH}_{\mathrm{a}} \mathrm{H}_{\mathrm{b}}\right)$, $2.96\left(\mathrm{dd}, J=12.8,6.8 \mathrm{~Hz}, 1 \mathrm{H}, \mathrm{CHCH}_{\mathrm{a}} \mathrm{H}_{\mathrm{b}}\right), 2.41\left(\mathrm{~s}, 3 \mathrm{H}, \mathrm{NCH}_{3}\right), 1.76$ (br s, $\left.3 \mathrm{H}, \mathrm{NH}+\mathrm{NH}_{2}\right), 1.67\left(\mathrm{~s}, 9 \mathrm{H}, \mathrm{OC}\left(\mathrm{CH}_{3}\right)_{3}\right)$.

${ }^{13} \mathrm{C}$ NMR $\left(100 \mathrm{MHz}, \mathrm{CDCl}_{3}\right): \delta=149.23,136.56,128.20,125.69$, $123.79,120.75,120.69,118.59,118.25,84.23,59.65,46.11,34.43$, $28.12(3 \mathrm{C})$.

MS (ESI): $m / z=370 / 368[\mathrm{M}+\mathrm{H}]^{+}$.

Anal. Calcd for $\mathrm{C}_{16} \mathrm{H}_{22} \mathrm{BrN}_{3} \mathrm{O}_{2}$ : C, 52.18; $\mathrm{H}, 6.02 ; \mathrm{N}, 11.41$. Found: C, 52.27; H, 6.07; N, 11.30 .

1-[5-Methoxy-(1-tert-butoxycarbonyl)-1H-indol-3-yl]- $N^{1}$-methylethane-1,2-diamine (6f)

Obtained by following General Procedure B.

Yield: $0.53 \mathrm{~g}$ (75\%); pale-yellow viscous oil; $R_{f}=0.34\left(\mathrm{CHCl}_{3}-\mathrm{MeOH}-\right.$ $\left.\mathrm{NH}_{3(\mathrm{aq})}, 10: 1: 0.02\right)$.

IR (film): 3325 br, 2976, 2836, 2794, 1730 s (CO), 1612, 1477, 1449, $1385,1256,1159,1074,855,803,732,656 \mathrm{~cm}^{-1}$.

${ }^{1} \mathrm{H}$ NMR (400 MHz, $\mathrm{CDCl}_{3}$ ): $\delta=8.02(\mathrm{~d}, J=9.0 \mathrm{~Hz}, 1 \mathrm{H}, 7-\mathrm{H}), 7.51(\mathrm{~s}$, $1 \mathrm{H}, 2-\mathrm{H}), 7.12(\mathrm{~d}, J=2.5 \mathrm{~Hz}, 1 \mathrm{H}, 4-\mathrm{H}), 6.92(\mathrm{dd}, J=9.0,2.5 \mathrm{~Hz}, 1 \mathrm{H}, 6-$ $\mathrm{H}), 3.84\left(\mathrm{~s}, 3 \mathrm{H}, \mathrm{OCH}_{3}\right), 3.81$ (dd J = 6.5, $\left.5.5 \mathrm{~Hz}, 1 \mathrm{H}, \mathrm{CHCH}_{\mathrm{a}} \mathrm{H}_{\mathrm{b}}\right), 3.09$ (dd, $J=12.9,5.5 \mathrm{~Hz}, 1 \mathrm{H}, \mathrm{CHCH}_{\mathrm{a}} \mathrm{H}_{\mathrm{b}}$ ), 2.97 (dd, $J=12.9,6.5 \mathrm{~Hz}, 1 \mathrm{H}, \mathrm{CH}-$ $\left.\mathrm{CH}_{\mathrm{a}} \mathrm{H}_{\mathrm{b}}\right), 2.42\left(\mathrm{~s}, 3 \mathrm{H}, \mathrm{NCH}_{3}\right), 1.91$ (br s, $\left.3 \mathrm{H}, \mathrm{NH}+\mathrm{NH}_{2}\right), 1.65(\mathrm{~s}, 9 \mathrm{H}$, $\left.\mathrm{OC}\left(\mathrm{CH}_{3}\right)_{3}\right)$.

${ }^{13} \mathrm{C}$ NMR $\left(100 \mathrm{MHz}, \mathrm{CDCl}_{3}\right): \delta=155.68,149.53,130.52,130.17$, 123.99, 120.33, 115.99, 112.89, 102.46, 83.42, 59.54, 55.73, 45.85, 34.32, $28.13(3 \mathrm{C})$.

MS (ESI): $m / z=320[\mathrm{M}+\mathrm{H}]^{+}$.

Anal. Calcd for $\mathrm{C}_{17} \mathrm{H}_{25} \mathrm{~N}_{3} \mathrm{O}_{3}$ : C, 63.93; H, 7.89; N, 13.16. Found: $\mathrm{C}$, 63.81; H, 7.96; N, 13.10 .

\section{1-[6-Chloro-(1-tert-butoxycarbonyl)-1H-indol-3-yl]- $N^{1}$-methyl-} ethane-1,2-diamine $(6 \mathrm{~g})$

Obtained by following General Procedure B.

Yield: $0.655 \mathrm{~g}$ (92\%); pale-yellow viscous oil; $R_{f}=0.32\left(\mathrm{CHCl}_{3}-\mathrm{MeOH}-\right.$ $\left.\mathrm{NH}_{3(\mathrm{aq})}, 10: 1: 0.02\right)$.

IR (film): 3366 br, 2978, 2937, 2796, 1737 s (CO), 1606, 1435, 1455, $1371,1252,11576,1087,812,767,733,595 \mathrm{~cm}^{-1}$. 
${ }^{1} \mathrm{H}$ NMR $\left(400 \mathrm{MHz}, \mathrm{CDCl}_{3}\right): \delta=8.18($ br s, $1 \mathrm{H}, 7-\mathrm{H}), 7.57(\mathrm{~d}, J=8.3 \mathrm{~Hz}$, $1 \mathrm{H}, 4-\mathrm{H}), 7.50$ (s, $1 \mathrm{H}, 2-\mathrm{H}), 7.16$ (dd, $J=8.3,2.0 \mathrm{~Hz}, 1 \mathrm{H}, 5-\mathrm{H}$ ), 3.80 (dd $J=6.7,5.3 \mathrm{~Hz}, 1 \mathrm{H}, \mathrm{CHCH}_{\mathrm{a}} \mathrm{H}_{\mathrm{b}}$ ), 3.06 (dd, $J=12.8,5.3 \mathrm{~Hz}, 1 \mathrm{H}, \mathrm{CHCH}_{\mathrm{a}} \mathrm{H}_{\mathrm{b}}$ ), $2.94\left(\mathrm{dd}, J=12.8,6.7 \mathrm{~Hz}, 1 \mathrm{H}, \mathrm{CHCH}_{\mathrm{a}} \mathrm{H}_{\mathrm{b}}\right), 2.38\left(\mathrm{~s}, 3 \mathrm{H}, \mathrm{NCH}_{3}\right), 2.01$ (br s, $\left.3 \mathrm{H}, \mathrm{NH}+\mathrm{NH}_{2}\right), 1.64\left(\mathrm{~s}, 9 \mathrm{H}, \mathrm{OC}\left(\mathrm{CH}_{3}\right)_{3}\right)$.

${ }^{13} \mathrm{C}$ NMR $\left(100 \mathrm{MHz}, \mathrm{CDCl}_{3}\right): \delta=149.24,136.24,130.48,127.83$, 123.91, 123.01, 120.61, 120.33, 115.67, 84.21, 59.46, 45.99, 34.37, $28.12(3 \mathrm{C})$.

MS (ESI): $m / z=326 / 324[\mathrm{M}+\mathrm{H}]^{+}$.

Anal. Calcd for $\mathrm{C}_{16} \mathrm{H}_{22} \mathrm{ClN}_{3} \mathrm{O}_{2}$ : C, 59.35; $\mathrm{H}, 6.85 ; \mathrm{N}, 12.98$. Found: $\mathrm{C}$, 59.27; H, 6.90; N, 12.91.

tert-Butyl 6-Bromo-3-\{2-[(6-bromo-1H-indol-3-yl)carbonyl]-1methyl-4,5-dihydro-1H-imidazol-5-yl\}-1H-indole-1-carboxylate (16a)

A solution of 3-acetyl-6-bromoindole $(0.2 \mathrm{~g}, 0.84 \mathrm{mmol})$ and $\mathrm{I}_{2}(0.23$ $\mathrm{g}, 0.92 \mathrm{mmol})$ in DMSO $(1.7 \mathrm{~mL})$ was heated for $2 \mathrm{~h}$ at $100^{\circ} \mathrm{C}$. The reaction mixture was cooled to $0-5{ }^{\circ} \mathrm{C}$ then treated with $\mathrm{NaHCO}_{3}(0.08$ $\mathrm{g}, 0.92 \mathrm{mmol})$ and a solution of diamine $6 \mathbf{e}(0.32 \mathrm{~g}, 0.86 \mathrm{mmol})$ in $\mathrm{MeCN}(8.5 \mathrm{~mL})$. After stirring for $0.5 \mathrm{~h}$ the reaction mixture was treated with NCS $(0.11 \mathrm{~g}, 0.86 \mathrm{mmol})$ under cooling $\left(0-5{ }^{\circ} \mathrm{C}\right)$, stirred for $20 \mathrm{~min}$, and left to stand overnight. The reaction mixture was diluted with $\mathrm{CHCl}_{3}(50 \mathrm{~mL})$, washed with saturated $\mathrm{NaHCO}_{3}(30 \mathrm{~mL})$ and $\mathrm{Na}_{2} \mathrm{~S}_{2} \mathrm{O}_{3}(30 \mathrm{~mL})$ solutions, $\mathrm{H}_{2} \mathrm{O}(5 \times 30 \mathrm{~mL})$, and concd $\mathrm{NaCl}$ solution $(30 \mathrm{~mL})$, and dried over anhydrous $\mathrm{Na}_{2} \mathrm{SO}_{4}$. The solvent was removed in vacuo and the solid was purified by chromatography on a column with silica gel $\left(\mathrm{CHCl}_{3}-\mathrm{MeOH}, 100: 1\right)$ to provide the title compound.

Yield: $0.388 \mathrm{~g}(77 \%)$; brownish amorphous solid; $R_{f}=0.53\left(\mathrm{CHCl}_{3}-\right.$ $\mathrm{MeOH}, 40: 1)$.

IR (film): 3119, 2978, 2869, 1742 s (CO), 1630, 1574, 1434, 1369, 1249, 1155, 1086, 980, 810, 689, $591 \mathrm{~cm}^{-1}$.

${ }^{1} \mathrm{H}$ NMR $\left(500 \mathrm{MHz}, \mathrm{CDCl}_{3}\right): \delta=12.14(\mathrm{br} \mathrm{s}, 1 \mathrm{H}, \mathrm{NH}), 8.41(\mathrm{br} \mathrm{s}, 1 \mathrm{H})$, $8.17(\mathrm{~d}, J=8.2 \mathrm{~Hz}, 1 \mathrm{H}), 7.98(\mathrm{~s}, 1 \mathrm{H}), 7.62(\mathrm{~s}, 1 \mathrm{H}), 7.44(\mathrm{~s}, 1 \mathrm{H}), 7.29-$ $7.41(\mathrm{~m}, 3 \mathrm{H}), 4.91\left(\mathrm{dd} J=12.2,10.4 \mathrm{~Hz}, 1 \mathrm{H}, \mathrm{CHCH}_{\mathrm{a}} \mathrm{H}_{\mathrm{b}}\right), 4.17$ (dd, $J=$ $\left.14.4,12.2 \mathrm{~Hz}, 1 \mathrm{H}, \mathrm{CHCH}_{\mathrm{a}} H_{\mathrm{b}}\right), 3.80(\mathrm{dd}, J=14.4,10.4 \mathrm{~Hz}, 1 \mathrm{H}, \mathrm{CH}-$ $\left.\mathrm{CH}_{\mathrm{a}} \mathrm{H}_{\mathrm{b}}\right), 2.81\left(\mathrm{~s}, 3 \mathrm{H}, \mathrm{NCH}_{3}\right), 1.70\left(\mathrm{~s}, 9 \mathrm{H}, \mathrm{OC}\left(\mathrm{CH}_{3}\right)_{3}\right)$.

${ }^{13} \mathrm{C}$ NMR $\left(100 \mathrm{MHz}, \mathrm{CDCl}_{3}\right): \delta=180.54,163.28,149.06,137.87$, 137.63, 136.93, 126.62, 126.42 (2C), 124.88, 124.57, 123.46, 120.55, $118.96,118.93,118.39,117.63,115.60,115.24,84.82,61.00,58.76$, 31.86, $28.16(3 C)$.

MS (ESI): $m / z=602 / 601 / 600[\mathrm{M}+\mathrm{H}]^{+}$.

Anal. Calcd for $\mathrm{C}_{26} \mathrm{H}_{24} \mathrm{Br}_{2} \mathrm{~N}_{4} \mathrm{O}_{3}$ : C, 52.02; $\mathrm{H}, 4.03 ; \mathrm{N}, 9.33$. Found: $\mathrm{C}$, $52.21 ; \mathrm{H}, 4.11 ; \mathrm{N}, 9.21$.

\section{tert-Butyl 3-[2-(1H-Indol-3-ylcarbonyl)-1-methyl-4,5-dihydro- $1 H$-imidazol-5-yl]-1H-indole-1-carboxylate (16b)}

The compound was prepared as described above for $\mathbf{1 6 a}$.

Yield: 0.290 (79\%); light-brown amorphous solid; $R_{f}=0.46\left(\mathrm{CHCl}_{3}-\right.$ $\mathrm{MeOH}, 40: 1)$.

IR (film): 3105 br, 2979, 2932, 1737 s (CO), 1640, 1607, 1514, 1451, $1369,1240,1154,1091,749,589 \mathrm{~cm}^{-1}$.

${ }^{1} \mathrm{H} \mathrm{NMR}\left(400 \mathrm{MHz}, \mathrm{CDCl}_{3}\right): \delta=12.40$ (br s, $\left.1 \mathrm{H}, \mathrm{NH}\right), 8.42(\mathrm{~d}, J=7.9 \mathrm{~Hz}$, $1 \mathrm{H}), 8.21(\mathrm{~d}, J=8.3 \mathrm{~Hz}, 1 \mathrm{H}), 7.88(\mathrm{~s}, 1 \mathrm{H}), 7.58-7.67(\mathrm{~m}, 2 \mathrm{H}), 7.34-$ $7.41(\mathrm{~m}, 1 \mathrm{H}), 7.20-7.29(\mathrm{~m}, 3 \mathrm{H}), 7.13-7.19(\mathrm{~m}, 1 \mathrm{H}), 4.82(\mathrm{dd} J=11.6$, $\left.10.6 \mathrm{~Hz}, 1 \mathrm{H}, \mathrm{CHCH}_{\mathrm{a}} \mathrm{H}_{\mathrm{b}}\right), 4.15$ (dd, $\left.J=14.5,11.6 \mathrm{~Hz}, 1 \mathrm{H}, \mathrm{CHCH}_{\mathrm{a}} \mathrm{H}_{\mathrm{b}}\right), 3.88$ (dd, $\left.J=14.5,10.6 \mathrm{~Hz}, 1 \mathrm{H}, \mathrm{CHCH}_{\mathrm{a}} \mathrm{H}_{\mathrm{b}}\right), 2.83\left(\mathrm{~s}, 3 \mathrm{H}, \mathrm{NCH}_{3}\right), 1.72(\mathrm{~s}, 9 \mathrm{H}$, $\left.\mathrm{OC}\left(\mathrm{CH}_{3}\right)_{3}\right)$.
${ }^{13} \mathrm{C}$ NMR $\left(100 \mathrm{MHz}, \mathrm{CDCl}_{3}\right): \delta=180.93,163.58,149.51,137.72$, $137.20,136.18,127.99,125.79,124.95,124.30,123.97,123.08$, $123.05,122.16,119.58,118.71,115.69,115.58,112.29,84.13,61.08$, $58.78,31.86,28.19(3 \mathrm{C})$.

MS (ESI): $m / z=443[\mathrm{M}+\mathrm{H}]^{+}$.

Anal. Calcd for $\mathrm{C}_{26} \mathrm{H}_{26} \mathrm{~N}_{4} \mathrm{O}_{3}$ : C, 70.57; H, 5.92; N, 12.66. Found: C, $70.41 ; \mathrm{H}, 5.84 ; \mathrm{N}, 12.55$.

tert-Butyl 6-Bromo-3-[5-(6-bromo-1H-indol-3-yl)-1-methyl-6oxo-1,2,3,6-tetrahydropyrazin-2-yl]-1H-indole-1-carboxylate (22a)

A solution of diamine $\mathbf{6 e}(0.26 \mathrm{~g}, 0.70 \mathrm{mmol})$ in EtOH $(2.6 \mathrm{~mL})$ at $0-$ $5{ }^{\circ} \mathrm{C}$ was treated portionwise with acid chloride 18a $(0.20 \mathrm{~g}, 0.7$ $\mathrm{mmol}$ ) over a period of $10 \mathrm{~min}$ and stirred for $15 \mathrm{~min}$ at r.t. Anhydrous AcONa ( $57.4 \mathrm{mg}, 0.7 \mathrm{mmol})$ and $\mathrm{AcOH}(0.26 \mathrm{~mL})$ were then added and the reaction mixture was heated at reflux for $1.5 \mathrm{~h}$. After cooling, the mixture was diluted with EtOAc $(25 \mathrm{~mL})$, washed with saturated $\mathrm{NaHCO}_{3}(20 \mathrm{~mL})$ and concd $\mathrm{NaCl}(10 \mathrm{~mL})$ solutions, and dried over anhydrous $\mathrm{Na}_{2} \mathrm{SO}_{4}$. The solvent was removed in vacuo and the residue was purified by chromatography on a column with silica gel (toluene-EtOAc, 3:1) to provide the title compound.

Yield: $0.302 \mathrm{~g}$ (72\%); pale-yellow amorphous solid; $R_{f}=0.49$ (tolueneEtOAc, 2:1).

IR (film): 3151 br, 2976, 2932, 1740 s (CO), 1650, 1590, 1434, 1369, $1252,1154,1088,809,766,731,589 \mathrm{~cm}^{-1}$.

${ }^{1} \mathrm{H} \mathrm{NMR}\left(600 \mathrm{MHz}, \mathrm{CDCl}_{3}\right): \delta=8.89(\mathrm{br} \mathrm{s}, 1 \mathrm{H}, \mathrm{NH}), 8.47(\mathrm{~d}, J=2.6 \mathrm{~Hz}$, $1 \mathrm{H}), 8.35(\mathrm{br} \mathrm{s}, 1 \mathrm{H}), 8.27(\mathrm{~d}, J=8.8 \mathrm{~Hz}, 1 \mathrm{H}), 7.54(\mathrm{~d}, J=1.8 \mathrm{~Hz}, 1 \mathrm{H})$, $7.45(\mathrm{~d}, J=8.4 \mathrm{~Hz}, 1 \mathrm{H}), 7.42(\mathrm{br} \mathrm{s}, 1 \mathrm{H}), 7.40(\mathrm{dd}, J=8.4,1.8 \mathrm{~Hz}, 1 \mathrm{H})$, 7.28 (dd, $J=8.8,1.8 \mathrm{~Hz}, 1 \mathrm{H}), 4.96\left(\mathrm{dd} J=5.9,5.5 \mathrm{~Hz}, 1 \mathrm{H}, \mathrm{CHCH}_{\mathrm{a}} \mathrm{H}_{\mathrm{b}}\right.$ ), $4.38\left(\mathrm{dd}, J=16.5,5.9 \mathrm{~Hz}, 1 \mathrm{H}, \mathrm{CHCH}_{\mathrm{a}} H_{\mathrm{b}}\right), 4.31(\mathrm{dd}, J=16.5,5.5 \mathrm{~Hz}, 1 \mathrm{H}$, $\left.\mathrm{CHCH}_{\mathrm{a}} \mathrm{H}_{\mathrm{b}}\right), 3.10\left(\mathrm{~s}, 3 \mathrm{H}, \mathrm{NCH}_{3}\right), 1.60\left(\mathrm{~s}, 9 \mathrm{H}, \mathrm{OC}\left(\mathrm{CH}_{3}\right)_{3}\right)$.

${ }^{13} \mathrm{C}$ NMR $\left(125 \mathrm{MHz}, \mathrm{CDCl}_{3}\right): \delta=157.84,157.21,148.91,136.90$, $136.52,131.99,127.18,126.25,125.22,124.65,124.54,124.10$, 119.98, 118.94, 118.79, 117.00, 116.40, 114.11, 112.13, 84.90, 53.31, $51.87,33.00,28.00$ (3C).

MS (ESI): $m / z=602 / 601 / 600[\mathrm{M}+\mathrm{H}]^{+}$.

Anal. Calcd for $\mathrm{C}_{26} \mathrm{H}_{24} \mathrm{Br}_{2} \mathrm{~N}_{4} \mathrm{O}_{3}$ : C, 52.02; $\mathrm{H}, 4.03 ; \mathrm{N}, 9.33$. Found: $\mathrm{C}$, 52.24; $\mathrm{H}, 4.11 ; \mathrm{N}, 9.24$.

tert-Butyl 3-[5-(1H-Indol-3-yl)-1-methyl-6-oxo-1,2,3,6-tetrahydropyrazin-2-yl]-1H-indole-1-carboxylate (22b)

The compound was prepared as described for 22a.

Yield: $0.214 \mathrm{~g}$ (69 \%); light-brown amorphous solid; $R_{f}=0.45$ (toluene-EtOAc, 2:1).

IR (film): 3270 br, 2976, 2931, 1736 s (CO), 1650, 1590, 1453, 1371, $1310,1256,1155,1088,852,746,591 \mathrm{~cm}^{-1}$.

${ }^{1} \mathrm{H} \mathrm{NMR}\left(400 \mathrm{MHz}, \mathrm{CDCl}_{3}\right): \delta=8.88(\mathrm{br} \mathrm{s}, 1 \mathrm{H}, \mathrm{NH}), 8.54(\mathrm{~d}, J=2.6 \mathrm{~Hz}$, $1 \mathrm{H}), 8.43(\mathrm{~d}, J=8.7 \mathrm{~Hz}, 1 \mathrm{H}), 8.14(\mathrm{~d}, J=8.0 \mathrm{~Hz}, 1 \mathrm{H}), 7.61(\mathrm{~d}, J=$ $7.7 \mathrm{~Hz}, 1 \mathrm{H}), 7.45(\mathrm{~s}, 1 \mathrm{H}), 7.33-7.42(\mathrm{~m}, 2 \mathrm{H}), 7.25-7.31(\mathrm{~m}, 1 \mathrm{H})$, 7.17-7.25 (m, $2 \mathrm{H}), 4.98$ (dd, $\left.J=5.9,5.4 \mathrm{~Hz}, 1 \mathrm{H}, \mathrm{CHCH}_{\mathrm{a}} \mathrm{H}_{\mathrm{b}}\right), 4.46$ (dd, $\left.J=16.5,5.9 \mathrm{~Hz}, 1 \mathrm{H}, \mathrm{CHCH}_{\mathrm{a}} \mathrm{H}_{\mathrm{b}}\right), 4.31(\mathrm{dd}, J=16.5,5.4 \mathrm{~Hz}, 1 \mathrm{H}, \mathrm{CH}-$ $\left.\mathrm{CH}_{\mathrm{a}} \mathrm{H}_{\mathrm{b}}\right), 3.13\left(\mathrm{~s}, 3 \mathrm{H}, \mathrm{NCH}_{3}\right), 1.60\left(\mathrm{~s}, 9 \mathrm{H}, \mathrm{OC}\left(\mathrm{CH}_{3}\right)_{3}\right)$.

${ }^{13} \mathrm{C}$ NMR $\left(100 \mathrm{MHz}, \mathrm{CDCl}_{3}\right): \delta=158.07,157.29,149.36,136.09$, 135.87, 132.13, 128.44, 126.23, 124.93, 124.18, 122.94 (2C), 122.70, $121.57,118.93,117.00,115.67,111.94,111.19,84.20,53.45,51.63$, 32.96, $28.06(3 \mathrm{C})$.

MS (ESI): $m / z=443[\mathrm{M}+\mathrm{H}]^{+}$. 
Anal. Calcd for $\mathrm{C}_{26} \mathrm{H}_{26} \mathrm{~N}_{4} \mathrm{O}_{3}$ : C, 70.57; H, 5.92; N, 12.66. Found: $\mathrm{C}$, $70.71 ; \mathrm{H}, 5.99 ; \mathrm{N}, 12.60$.

\section{Removal of the Boc Protecting Group; General Procedure}

A suspension of the substrate $(0.43 \mathrm{mmol})$ in $\mathrm{CH}_{2} \mathrm{Cl}_{2}(2 \mathrm{~mL})$ at $0-5{ }^{\circ} \mathrm{C}$ was treated with TFA $(0.4 \mathrm{~mL}, 5.2 \mathrm{mmol})$ in four portions, stirred at the same temperature for $20 \mathrm{~min}$, left overnight, and evaporated in vacuo. The residue was dissolved in EtOAc $(40 \mathrm{~mL})$, washed with saturated $\mathrm{NaHCO}_{3}(2 \times 20 \mathrm{~mL})$ and concd $\mathrm{NaCl}(20 \mathrm{~mL})$ solutions, and dried over anhydrous $\mathrm{Na}_{2} \mathrm{SO}_{4}$. The solvent was removed in vacuo and the residue was purified by chromatography on a column with silica gel.

\section{(6-Bromo-1H-indol-3-yl)[5-(6-bromo-1H-indol-3-yl)-1-methyl-} 4,5-dihydro-1H-imidazol-2-yl]methanone (5a)

Yield: $0.210 \mathrm{~g}$ (98\%); light-beige solid; $\mathrm{mp} 247-249{ }^{\circ} \mathrm{C}$ (dec.) (EtOAc); $R_{f}=0.36\left(\mathrm{CHCl}_{3}-\mathrm{MeOH}, 20: 1\right)$.

IR (film): 3426, 3119, 3012, 2850, 1637, 1569, 1513, 1450, 1265, $1085,986,822,793,692,600 \mathrm{~cm}^{-1}$.

${ }^{1} \mathrm{H}$ NMR (400 MHz, DMSO- $d_{6}$ ): $\delta=12.30$ (br s, $\left.1 \mathrm{H}, 1^{\prime}-\mathrm{NH}\right), 11.20$ (br s, $\left.1 \mathrm{H}, 1^{\prime \prime}-\mathrm{NH}\right), 8.51$ (s, $\left.1 \mathrm{H}, 2^{\prime}-\mathrm{H}\right), 8.17$ (d, $\left.J=8.4 \mathrm{~Hz}, 1 \mathrm{H}, 4^{\prime}-\mathrm{H}\right), 7.74$ (d, $\left.J=1.6 \mathrm{~Hz}, 1 \mathrm{H}, 7^{\prime}-\mathrm{H}\right), 7.59\left(\mathrm{~d}, J=1.6 \mathrm{~Hz}, 1 \mathrm{H}, 7^{\prime \prime}-\mathrm{H}\right), 7.53(\mathrm{~d}, J=8.4 \mathrm{~Hz}$, $1 \mathrm{H}, 4^{\prime \prime}-\mathrm{H}$ ), 7.45 (d, J = 2.4 Hz, $1 \mathrm{H}, 2^{\prime \prime}-\mathrm{H}$ ), 7.40 (dd, J = 8.4, $1.6 \mathrm{~Hz}, 1 \mathrm{H}$, $\left.5^{\prime}-\mathrm{H}\right), 7.14$ (dd, $\left.J=8.4,1.6 \mathrm{~Hz}, 1 \mathrm{H}, 5^{\prime \prime}-\mathrm{H}\right), 4.86$ (dd $J=11.2,10.3 \mathrm{~Hz}$, $\left.1 \mathrm{H}, \mathrm{CHCH}_{\mathrm{a}} \mathrm{H}_{\mathrm{b}}\right), 4.26\left(\mathrm{dd}, J=15.1,11.2 \mathrm{~Hz}, 1 \mathrm{H}, \mathrm{CHCH}_{\mathrm{a}} H_{\mathrm{b}}\right), 3.80(\mathrm{dd}, J=$ 15.1, $\left.10.3 \mathrm{~Hz}, 1 \mathrm{H}, \mathrm{CHCH}_{\mathrm{a}} \mathrm{H}_{\mathrm{b}}\right), 2.67\left(\mathrm{~s}, 3 \mathrm{H}, \mathrm{NCH}_{3}\right)$.

${ }^{13} \mathrm{C}$ NMR (100 MHz, DMSO- $\left.d_{6}\right): \delta=182.24,162.09,139.09,137.80$, 137.60, 125.27 (2C), 124.65, 124.43, 122.90, 121.67, 120.56, 115.82, $115.23,114.80,114.40,114.13,113.88,60.54$ (2C), 31.69.

${ }^{1} \mathrm{H}$ NMR (400 MHz, acetone- $d_{6}$ ): $\delta=11.37$ (br s, $\left.1 \mathrm{H}, 1^{\prime}-\mathrm{NH}\right), 10.42$ (br s, $1 \mathrm{H}, 1^{\prime \prime}-\mathrm{NH}$ ), 8.68 (s, $1 \mathrm{H}, 2^{\prime}-\mathrm{H}$ ), 8.32 (d, J = 8.5 Hz, $\left.1 \mathrm{H}, 4^{\prime}-\mathrm{H}\right), 7.76$ (d, $\left.J=1.6 \mathrm{~Hz}, 1 \mathrm{H}, 7^{\prime}-\mathrm{H}\right), 7.65\left(\mathrm{~d}, J=1.6 \mathrm{~Hz}, 1 \mathrm{H}, 7^{\prime \prime}-\mathrm{H}\right), 7.63$ (d, $J=$ $\left.8.5 \mathrm{~Hz}, 1 \mathrm{H}, 4^{\prime \prime}-\mathrm{H}\right), 7.45$ (d, $\left.J=2.1 \mathrm{~Hz}, 1 \mathrm{H}, 2^{\prime \prime}-\mathrm{H}\right), 7.41$ (dd, $J=8.5$, $1.7 \mathrm{~Hz}, 1 \mathrm{H}, 5^{\prime}-\mathrm{H}$ ), 7.17 (dd, $J=8.5,1.7 \mathrm{~Hz}, 1 \mathrm{H}, 5^{\prime \prime}-\mathrm{H}$ ), 4.90 (dd $J=11.3$, $10.3 \mathrm{~Hz}, 1 \mathrm{H}, \mathrm{CHCH}_{\mathrm{a}} \mathrm{H}_{\mathrm{b}}$ ), 4.31 (dd, $\left.J=15.3,11.3 \mathrm{~Hz}, 1 \mathrm{H}, \mathrm{CHCH}_{\mathrm{a}} \mathrm{H}_{\mathrm{b}}\right), 3.91$ (dd, $\left.J=15.3,10.3 \mathrm{~Hz}, 1 \mathrm{H}, \mathrm{CHCH}_{\mathrm{a}} \mathrm{H}_{\mathrm{b}}\right), 2.82\left(\mathrm{~s}, 3 \mathrm{H}, \mathrm{NCH}_{3}\right)$.

${ }^{13} \mathrm{C}$ NMR $\left(100 \mathrm{MHz}\right.$, acetone- $\left.d_{6}\right): \delta=183.28,163.42,139.61,139.36$, $138.78,126.40,126.31,125.93,125.78,124.34,123.13,121.83$, $117.27,116.72,116.13,115.88,115.83,115.59,62.40,61.88,32.51$.

MS (ESI): $m / z=502 / 501 / 500[\mathrm{M}+\mathrm{H}]^{+}$.

Anal. Calcd for $\mathrm{C}_{21} \mathrm{H}_{16} \mathrm{Br}_{2} \mathrm{~N}_{4} \mathrm{O}$ : C, 50.43; $\mathrm{H}, 3.22 ; \mathrm{N}, 11.20$. Found: $\mathrm{C}$, 50.49; $\mathrm{H}, 3.26 ; \mathrm{N}, 11.18$.

\section{H-Indol-3-yl[5-(1H-indol-3-yl)-1-methyl-4,5-dihydro-1H-imid- azol-2-yl]methanone (5b)}

Yield: $0.139 \mathrm{~g}$ (94\%); light-brown amorphous solid; $R_{f}=0.33\left(\mathrm{CHCl}_{3}-\right.$ $\mathrm{MeOH}, 20: 1)$.

IR (film): 3360 br, 3059, 2919, 1620, 1580, 1518, 1442, 1241, 1085 , $977,858,743,642 \mathrm{~cm}^{-1}$.

${ }^{1} \mathrm{H}$ NMR $\left(400 \mathrm{MHz}\right.$, DMSO- $\left.d_{6}\right): \delta=12.22$ (br s, $\left.1 \mathrm{H}, 1^{\prime}-\mathrm{NH}\right), 11.08$ (br s, $1 \mathrm{H}, 1$ "-NH), $8.50(\mathrm{~s}, 1 \mathrm{H}), 8.24-8.29(\mathrm{~m}, 1 \mathrm{H}), 7.61(\mathrm{~d}, J=8.0 \mathrm{~Hz}, 1 \mathrm{H})$, 7.52-7.58 (m, $1 \mathrm{H}), 7.37-7.44$ (m, $2 \mathrm{H}), 7.22-7.32$ (m, $2 \mathrm{H}), 7.08-7.15$ $(\mathrm{m}, 1 \mathrm{H}), 6.97-7.04(\mathrm{~m}, 1 \mathrm{H}), 4.88\left(\mathrm{dd} J=11.3,10.2 \mathrm{~Hz}, 1 \mathrm{H}, \mathrm{CHCH}_{\mathrm{a}} \mathrm{H}_{\mathrm{b}}\right)$, $4.26\left(\mathrm{dd}, J=14.8,11.3 \mathrm{~Hz}, 1 \mathrm{H}, \mathrm{CHCH}_{\mathrm{a}} H_{\mathrm{b}}\right), 3.86(\mathrm{dd}, J=14.8,10.2 \mathrm{~Hz}$, $\left.1 \mathrm{H}, \mathrm{CHCH}_{\mathrm{a}} \mathrm{H}_{\mathrm{b}}\right), 2.68\left(\mathrm{~s}, 3 \mathrm{H}, \mathrm{NCH}_{3}\right)$.

${ }^{13} \mathrm{C}$ NMR $\left(100 \mathrm{MHz}\right.$, DMSO- $\left.d_{6}\right): \delta=182.32,162.48,138.42,137.02$, 137.79, 125.62, 125.50, 124.34, 123.46, 122.52, 121.36 (2C), 118.94, $118.83,115.01,113.41,112.60,111.92,60.83,60.36,31.73$.
MS (ESI): $m / z=343[\mathrm{M}+\mathrm{H}]^{+}$.

Anal. Calcd for $\mathrm{C}_{21} \mathrm{H}_{18} \mathrm{~N}_{4} \mathrm{O}$ : C, 73.67; $\mathrm{H}, 5.30 ; \mathrm{N}, 16.36$. Found: $\mathrm{C}, 73.48$; H, 5.23; N, 16.28.

\section{Topsentin C (17a)}

Yield: $0.214 \mathrm{~g}(99 \%)$; pale-yellow solid; $\mathrm{mp} 179-181{ }^{\circ} \mathrm{C}$ (dec.) $(i-$ PrOH); $R_{f}=0.18\left(\mathrm{CHCl}_{3}\right)$.

IR (film): 3404, 3208, 2924, 2853, 1647, 1592, 1524, 1440, 1397, 1340, 1290, 1231, 1111, 1049, 947, 895, 852, 793, 734, 647, $570 \mathrm{~cm}^{-1}$. ${ }^{1} \mathrm{H}$ NMR (400 MHz, DMSO- $d_{6}$ ): $\delta=11.62$ (br s, 1 H, $\left.1^{\prime}-\mathrm{NH}\right), 11.14$ (br s, $\left.1 \mathrm{H}, 1^{\prime \prime}-\mathrm{NH}\right), 8.46$ (d, $\left.J=2.4 \mathrm{~Hz}, 1 \mathrm{H}, 2^{\prime}-\mathrm{H}\right), 8.22\left(\mathrm{~d}, J=8.6 \mathrm{~Hz}, 1 \mathrm{H}, 4^{\prime}-\right.$ H), 7.49-7.71 (m, 3 H, 7'-H + 7"-H + 4"-H), 7.03-7.25 (m, 3 H, 2"-H + $5^{\prime}-\mathrm{H}+5^{\prime \prime}-\mathrm{H}$ ), 5.11 (dd, $\left.J=5.3,4.8 \mathrm{~Hz}, 1 \mathrm{H}, \mathrm{CHCH}_{\mathrm{a}} \mathrm{H}_{\mathrm{b}}\right), 4.29$ (dd, $J=16.5$, $4.8 \mathrm{~Hz}, 1 \mathrm{H}, \mathrm{CHCH}_{\mathrm{a}} \mathrm{H}_{\mathrm{b}}$ ), $4.18\left(\mathrm{dd}, J=16.5,5.3 \mathrm{~Hz}, 1 \mathrm{H}, \mathrm{CHCH}_{\mathrm{a}} \mathrm{H}_{\mathrm{b}}\right), 2.97$ (s, $3 \mathrm{H}, \mathrm{NCH}_{3}$ ).

${ }^{13} \mathrm{C}$ NMR (100 MHz, DMSO- $\left.d_{6}\right): \delta=157.08,156.69,137.42,137.04$, $132.77,125.09,124.64,124.52,124.10,123.21,121.83,120.33$, $114.70,114.35,114.20$ (2C), 112.12, 111.02, 52.46, 52.28, 32.36 .

${ }^{1} \mathrm{H}$ NMR (400 MHz, acetone- $d_{6}$ ): $\delta=10.70$ (br s, $1 \mathrm{H}, 1$ '-NH), 10.34 (br s, $1 \mathrm{H}, 1^{\prime \prime}-\mathrm{NH}$ ), 8.62 (d, $J=2.75 \mathrm{~Hz}, 1 \mathrm{H}, 2^{\prime}-\mathrm{H}$ ), 8.37 (d, $J=8.6 \mathrm{~Hz}$, $\left.1 \mathrm{H}, 4^{\prime}-\mathrm{H}\right), 7.69$ (d, $\left.J=8.6 \mathrm{~Hz}, 1 \mathrm{H}, 4^{\prime \prime}-\mathrm{H}\right), 7.66\left(\mathrm{~d}, J=1.8 \mathrm{~Hz}, 1 \mathrm{H}, 7^{\prime}-\mathrm{H}\right)$, 7.63 (d, $\left.J=1.7 \mathrm{~Hz}, 1 \mathrm{H}, 7^{\prime \prime}-\mathrm{H}\right), 7.17-7.23$ (m, $\left.3 \mathrm{H}, 2^{\prime \prime}-\mathrm{H}+5^{\prime}-\mathrm{H}+5^{\prime \prime}-\mathrm{H}\right)$, 5.15 (dd $\left.J=5.5,5.2 \mathrm{~Hz}, 1 \mathrm{H}, \mathrm{CHCH}_{\mathrm{a}} \mathrm{H}_{\mathrm{b}}\right), 4.41(\mathrm{dd}, J=16.5,5.2 \mathrm{~Hz}, 1 \mathrm{H}$, $\left.\mathrm{CHCH}_{\mathrm{a}} \mathrm{H}_{\mathrm{b}}\right), 4.26\left(\mathrm{dd}, J=16.5,5.5 \mathrm{~Hz}, 1 \mathrm{H}, \mathrm{CHCH}_{\mathrm{a}} \mathrm{H}_{\mathrm{b}}\right), 3.04(\mathrm{~s}, 3 \mathrm{H}$, $\mathrm{NCH}_{3}$ ).

${ }^{13} \mathrm{C}$ NMR (100 MHz, acetone- $\left.d_{6}\right): \delta=158.47,158.06,138.86,138.34$, $133.67,126.67,126.07,125.49,125.39,124.40,123.30,121.19$, 116.17, 115.82, 115.53, 115.09, 113.93, 113.05, 54.07, 53.54, 32.77.

MS (ESI): $m / z=502 / 501 / 500[\mathrm{M}+\mathrm{H}]^{+}$.

Anal. Calcd for $\mathrm{C}_{21} \mathrm{H}_{16} \mathrm{Br}_{2} \mathrm{~N}_{4} \mathrm{O}$ : C, 50.43; H, 3.22; N, 11.20. Found: C, 50.55; H, 3.25; N, 11.16.

\section{3,6-Di-1H-Indol-3-yl-1-methyl-5,6-dihydropyrazin-2(1H)-one (17b)}

Yield: $0.141 \mathrm{~g}(96 \%) ; R_{\mathrm{f}}=0.60\left(\mathrm{CHCl}_{3}-\mathrm{MeOH}, 15: 1\right)$.

IR (film): 3400, 3271, 3055, 2926, 1644, 1589, 1422, 1339, 1239, $1172,1100,1011,851,744 \mathrm{~cm}^{-1}$.

${ }^{1} \mathrm{H}$ NMR (600 MHz, DMSO- $d_{6}$ ): $\delta=11.53($ br s, $1 \mathrm{H}, \mathrm{NH}), 11.01$ (br s, $1 \mathrm{H}, \mathrm{NH}), 8.44(\mathrm{~d}, J=3.3 \mathrm{~Hz}, 1 \mathrm{H}), 8.29(\mathrm{~d}, J=8.3 \mathrm{~Hz}, 1 \mathrm{H}), 7.64(\mathrm{~d}, J=$ $8.3 \mathrm{~Hz}, 1 \mathrm{H}), 7.42(\mathrm{~d}, J=8.3 \mathrm{~Hz}, 1 \mathrm{H}), 7.36(\mathrm{~d}, J=8.3 \mathrm{~Hz}, 1 \mathrm{H}), 7.08-7.17$ $(\mathrm{m}, 2 \mathrm{H}), 7.07(\mathrm{~d}, J=2.5 \mathrm{~Hz}, 1 \mathrm{H}), 6.98-7.05(\mathrm{~m}, 2 \mathrm{H}), 5.11(\mathrm{dd}, J=5.8$, $\left.4.9 \mathrm{~Hz}, 1 \mathrm{H}, \mathrm{CHCH}_{\mathrm{a}} \mathrm{H}_{\mathrm{b}}\right), 4.30\left(\mathrm{dd}, J=16.5,4.9 \mathrm{~Hz}, 1 \mathrm{H}, \mathrm{CHCH}_{\mathrm{a}} \mathrm{H}_{\mathrm{b}}\right), 4.19$ (dd, $\left.J=16.5,5.8 \mathrm{~Hz}, 1 \mathrm{H}, \mathrm{CHCH}_{\mathrm{a}} \mathrm{H}_{\mathrm{b}}\right), 2.99\left(\mathrm{~s}, 3 \mathrm{H}, \mathrm{NCH}_{3}\right.$ ).

${ }^{13} \mathrm{C}$ NMR $\left(125 \mathrm{MHz}\right.$, DMSO- $\left.d_{6}\right): \delta=157.43,157.02,136.63,136.17$, $132.06,126.14,125.73,123.46,122.54,122.07,121.46,120.44$, $119.09,118.59,111.85$ (2C), 111.61, 111.06, 52.78, 52.40, 32.47.

MS (ESI): $m / z=343[\mathrm{M}+\mathrm{H}]^{+}$

Anal. Calcd for $\mathrm{C}_{21} \mathrm{H}_{18} \mathrm{~N}_{4} \mathrm{O}: \mathrm{C}, 73.67 ; \mathrm{H}, 5.30 ; \mathrm{N}, 16.36$. Found: C, 73.79; H, 5.34; N, 16.31 .

\section{Acknowledgment}

This work was supported by the Ministry of Education and Science of the Russian Federation (Agreement number 02.a03.21.0008) and by RFBR (research project No. 16-03-00190 a). 


\section{Supporting Information}

Supporting information for this article is available online at http://dx.doi.org/10.1055/s-0036-1588731.

\section{References}

(1) (a) Blunt, J. W.; Copp, B. R.; Keyzers, R. A.; Munro, M. H. G.; Prinsep, M. R. Nat. Prod. Rep. 2015, 32, 116. (b) Newman, D. J.; Cragg, G. M. J. Nat. Prod. 2012, 75, 311. (c) Skropeta, D. Nat. Prod. Rep. 2008, 25, 1131.

(2) Golantsov, N. E.; Festa, A. A.; Karchava, A. V.; Yurovskaya, M. A. Chem. Heterocycl. Compd. 2013, 49, 203.

(3) Morris, S. A.; Andersen, R. J. Tetrahedron 1990, 46, 715.

(4) (a) Gribble, G. W. Chem. Soc. Rev. 1999, 28, 335. (b) Gribble, G. W. Chemosphere 2003, 52, 289.

(5) For total syntheses of spongotines and topsentins, see: (a) Guinchard, X.; Vallée, Y.; Denis, J.-N. J. Org. Chem. 2007, 72, 3972. (b) Guinchard, X.; Vallée, Y.; Denis, J.-N. Org. Lett. 2007, 9, 3761. (c) Murai, K.; Morishita, M.; Nakatani, R.; Kubo, O.; Fujioka, H.; Kita, Y. J. Org. Chem. 2007, 27, 8947. (d) Mal, S. M.; Bohé, L.; Achab, S. Tetrahedron 2008, 64, 5904. (e) Miyake, F. Y.; Yakushijin, K.; Horne, D. A. Org. Lett. 2000, 2, 2121. (f) Kawasaki, I.; Katsuma, H.; Nakayama, Y.; Yamashita, M.; Ohta, S. Heterocycles 1998, 48, 1887. (g) Braekman, J. C.; Daloze, D.; Moussiaux, B.; Stoller, C.; Deneubourg, F. Pure Appl. Chem. 1989, 61, 509. (h) Tsujii, S.; Rinehart, K. L. J. Org. Chem. 1988, 53, 5446.

(6) For total syntheses of hamacanthins and dragmacidins, see: (a) Kouko, T.; Matsumura, K.; Kawasaki, T. Tetrahedron 2005, 2309. (b) Kawasaki, T.; Kouko, T.; Totsuka, H.; Hiramatsu, K. Tetrahedron Lett. 2003, 44, 8849. (c) Miyake, F. Y.; Yakushijin, K.; Horne, D. A. Org. Lett. 2002, 4, 941. (d) Jiang, B.; Yang, C.-G.; Wang, J. J. Org. Chem. 2002, 67, 1396. (e) Jiang, B.; Yang, C.-G.; Wang, J. J. Org. Chem. 2001, 66, 4865. (f) Zhang, F.; Wang, B.; Prasad, P.; Capon, R. J.; Jia, Y. Org. Lett. 2015, 17, 1529. (g) Jackson, J. J.; Kobayashi, H.; Steffens, S. D.; Zakarian, A. Angew. Chem. Int. Ed. 2015, 54, 9971. (h) Mandal, D.; Yamaguchi, A. D.; Yamaguchi, J.; Itami, K. J. Am. Chem. Soc. 2011, 133, 19660. (i) Tonsiengsom, F.; Miyake, F. Y.; Yakushijin, K.; Horne, D. A. Synthesis 2006, 49. (j) Garg, N. K.; Caspi, D. D.; Stoltz, B. M. J. Am. Chem. Soc. 2005, 127, 5970. (k) Garg, N. K.; Sarpong, R.; Stoltz, B. M. J. Am. Chem. Soc. 2002, 124, 13179. (l) Yang, C.-G.; Wang, J.; Tang, X.-X.; Jiang, B. Tetrahedron: Asymmetry 2002, 13, 383. (m) Kawasaki, T.; Enoki, H.; Matsumura, K.; Ohyama, M.; Inagawa, M.; Sakamoto, M. Org. Lett. 2000, 2, 3027. (n) Miyake, F. Y.; Yakushijin, K.; Horne, D. A. Org. Lett. 2000, 2, 3185. (o) Whitlock, C. R.; Cava, M. P. Tetrahedron Lett. 1994, 35, 371. (p) Jiang, B.; Smallheer, J. M.; Amaral-Ly, C.; Wuonola, M. A. J. Org. Chem. 1994, 59, 6823.
(7) (a) Kim, A.; Kim, M. J.; Noh, T. H.; Hong, J.; Liu, Y.; Wei, X.; Jung, J. H. Bioorg. Med. Chem. Lett. 2016, 26, 5013. (b) Veale, C. G. L.; Zoraghi, R.; Young, R. M.; Morrison, J. P.; Pretheeban, M.; Lobb, K. A.; Reiner, N. E.; Andersen, R. J.; Davies-Coleman, M. T. J. Nat. Prod. 2015, 78, 355. (c) Pinchuk, B.; Johannes, E.; Gul, S.; Schlosser, J.; Schaechtele, C.; Totzke, F.; Peifer, C. Marine Drugs 2013, 11, 3209.

(8) Hequet, A.; Burchak, O. N.; Jeanty, M.; Guinchard, X.; Le Pihive, E.; Maigre, L.; Bouhours, P.; Schneider, D.; Maurin, M.; Paris, J.M.; Denis, J.-N.; Jolivalt, C. ChemMedChem 2014, 9, 1534.

(9) Bulatova, N. N.; Suvorov, N. N. Chem. Heterocycl. Compd. 1969, 5, 602.

(10) Canoira, L.; Rodriguez, J. G.; Subirats, J. B.; Escario, J.-A.; Jimenez, I.; Martinez-Fernandez, A. R. Eur. J. Med. Chem. 1989, $24,39$.

(11) Mouhtaram, M.; Jung, L.; Stambach, J. F. Tetrahedron 1993, 49, 1391.

(12) (a) Imagawa, K.; Hata, E.; Yamada, T.; Mukaiyama, T. Chem. Lett. 1996, 4, 291. (b) O'Donovan, D. H.; Rozas, I. Tetrahedron Lett. 2012, 53, 4532.

(13) Robiette, R.; Tran, T.-V.; Cordi, A.; Tinant, B.; MarchandBrynaert, J. Synthesis 2010, 3138.

(14) (a) Beshara, C. S.; Hall, A.; Jenkins, R. L.; Jones, K. L.; Jones, T. C.; Killeen, N. M.; Taylor, P. H.; Thomas, S. P.; Tomkinson, N. C. O. Org. Lett. 2005, 7, 5729. (b) Smithen, D. A.; Mathews, C. J.; Tomkinson, N. C. O. Org. Biomol. Chem. 2012, 10, 3756.

(15) (a) Erdik, E. Tetrahedron 2004, 60, 8747. (b) Grohmann, C.; Wang, H.; Glorius, F. Org. Lett. 2013, 15, 3014.

(16) Marmer, W. N.; Maerker, G. J. Org. Chem. 1972, 37, 3520.

(17) Wang, H.; Glorius, F. Angew. Chem. Int. Ed. 2012, 51, 7318.

(18) Murai, K.; Takaichi, N.; Takahara, Y.; Fukushima, S.; Fujioka, H. Synthesis 2010, 520.

(19) (a) Battini, N.; Padala, A. K.; Mupparapu, N.; Vishwakarma, R. A.; Ahmed, Q. N. RSC Adv. 2014, 4, 26258. (b) Xiang, J.; Wang, J.; Wang, M.; Meng, X.; Wu, A. Tetrahedron 2014, 70, 7470.

(20) Gunasekera, S. P.; McCarthy, P. J.; Kelly-Borges, M. J. Nat. Prod. 1994, 57, 1437.

(21) We did not observe the formation of imidazoline derivatives $\mathbf{1 6 a}, \mathbf{b}$ in this reaction. It should also be noted that, in our experiments, compound $\mathbf{5 a}, \mathbf{b}$ did not transform into the corresponding dihydropyrazinone derivatives $\mathbf{1 7 a}, \mathbf{b}$ upon heating in protic or aprotic solvents in the presence of acid (AcOH, PTSA, TFA, $\mathrm{HCl}$ ).

(22) NMR ${ }^{1} \mathrm{H}$ spectra of natural topsentin $\mathrm{C}$ and compound 17a are identical. There are differences in chemical shifts of several low intensity signals of tertiary carbon atoms in the ${ }^{13} \mathrm{C}$ spectra. It is clear that due to the very small amount of isolated topsentin C, background signals or admixtures masked these low peaks in the spectrum of the natural compound.

(23) Cassel, S.; Casenave, B.; Déléris, G.; Latxague, L.; Rollin, P. Tetrahedron 1998, 54, 8515 